Geophysical and Astrophysical Fluid Dynamics

Vol. 00, No. 00, 00 Month 2019, 1-30

\title{
The alignment of two three-dimensional quasi-geostrophic vortices
}

\author{
Jean N. Reinaud $\dagger^{*}$ and Xavier Carton $\ddagger$ \\ † Mathematical Institute, North Haugh, St Andrews, KY169SS, Scotland \\ ‡ LOPS/IUEM UBO, Technopôle Brest-Iroise 29280 Plouzané, France \\ (14 September 2017)
}

\begin{abstract}
We consider the interaction between two quasi-geostrophic vortices of height-to-width aspect ratio $h / r$, lying at two different vertical levels. We investigate whether such structures naturally align. In the case the vortices occupy distinct yet contiguous vertical levels, such an alignment can contribute to the growth in volume of oceanic mesoscale vortices. The other growth mechanism is the merger of vortices sharing common vertical levels. We show that there exist titled equilibrium states where vortices nearly align slantwise. Most equilibria for prolate vortices $(h / r>1)$ are stable apart in a very narrow region of the parameter space. The instability is however normally non-destructive. Pairs of oblate vortices may also be in an unstable equilibria if they are moderately offset in the horizontal direction. In this case, the instability may result in the shedding of filamentary potentially vorticity away from the vortices. This shedding of potential vorticity may result in the further alignment of the main structures.
\end{abstract}

Keywords: Vortex dynamics; Vortex alignment; Quasi-geostrophy

\section{Introduction}

Large scale oceanic motions are strongly influenced by the background planetary rotation and the stable density stratification of the medium. When these effects are dominant, the flow evolution can be accurately studied within the framework the quasi-geostrophic (QG) model. In this model, both geostrophic and hydrostatic balances approximatively hold. The vertical velocity and the vertical advection are then negligible. In the QG model, the velocity field can be deduced from the spatial distribution of the materially conversed QG potential vorticity anomaly (hereinafter referred to as PV for simplicity).

Vortices can be defined as contiguous regions of PV. Vortices are ubiquitous features in the oceans. Recent estimates by Zhang et al. (2014) suggest that an important part of the mass transport in the oceans can be attributed to mesoscale vortices. Mesoscale oceanic vortices are swirling masses of fluid having a horizontal radius in the approximate range $50-250 \mathrm{~km}$ which is typically roughly of the order of magnitude of the Rossby deformation radius.

Vortices do not evolve in isolation but they interact with other vortices. In particular, they can interact to form larger vortices. The formation of these larger vortices transfers energy towards large scales, in the so-called inverse energy cascade. The interaction also generates small scale structures and filaments contributing to direct energy cascade at small scales.

In this paper, we investigate the interaction between two QG vortices in a inbounded threedimensional fluid domain. There are two main interaction mechanisms which have been put forward to explain the growth in volume of the vortices. The first mechanism is vortex merger. Vortex merger has been extensively studied in the literature. In two dimensions, vortex merger provides a possible route for the classical inverse energy cascade observed two-dimensional turbulence. The merger of two-dimensional vortices has been studied in the pioneering works by

*Corresponding author: Email: jean.reinaud@st-andrews.ac.uk 
Overman II and Zabusky (1982), Melander et al. (1988), Waugh (1992), Dritschel and Waugh (1992) and many other authors. Vortices merge if they are separated by a distance smaller than a threshold called the critical merging distance. The merger can be associated with the emergence of an unstable mode for pairs of co-rotating vortices in mutual equilibrium, see Saffman and Szeto (1980). In a three-dimensional, rapidly rotating, continuously stratified fluid, vortex merger is possible provided the vortices share common vertical levels. This restriction is due to the absence of vertical motion in the QG model. The merger of threedimensional QG vortices has been studied by von Hardenberg et al. (2000), Dritschel (2002), Reinaud and Dritschel (2002, 2005), Bambrey et al. (2007), Özuğurlu et al. (2008). As in the two-dimensional case, the vortices may merge provided they are closer than a critical distance which corresponds to the onset of an unstable mode for an equilibrium.

The second mechanism to explain the growth in size of the vortices is the vertical alignment of vortices. Vertical alignment can occur when the vortices do not share any vertical level. In this case, the lack of vertical motion makes the merger impossible. Yet, two vortices can still form a large contiguous region of PV by aligning vertically. The process of alignment has been studied by Polvani (1991), Corréard and Carton (1998), Jones et al. (2009) in a twolayer flow. In a continuously stratified fluid alignment has been by Viera (1995), Sutyrin et al. (1998), Reasor and Montgomery (2001), Schecter et al. (2002), Reasor et al. (2004), Reasor and Montgomery (2015). Sutyrin et al. (1998) focused on the alignment of thin core vortices while the other studies focused on the alignment and axi-symmetrization of a single tilted columnar vortex. The orientation of a vortex, hence its vertical alignment is influenced by the vertical shear it is subjected to, see McKiver and Dritschel (2006). Alignment processes are also discussed in Ferrari and Paparella (2003), Haney et al. (2015), Itoh and Yasuda (2010), Tanahashi et al. (2001). Finally vertical alignment has been observed in geophysical turbulence by McWilliams (1989), McWilliams and Weiss (1994), in complex vortex interactions by Perrot et al. (2010), Sokolovskiy and Carton (2010), Viúdez (2010), Sokolvskiy and Verron (2014), Sokolovskiy et al. (2016) and in the oceans by Tychensky and Carton (1998) as well as in the laboratory experiments by Nof and Dewar (1994).

In this paper we focus on the alignment of two distinct, finite height-to-width vortices. In a similar set-up, Miyazaki et al. (2001) and Martinsen-Burrell et al. (2006) considered the problem of vortex merger and vortex alignment for pairs of co-rotating ellipsoidal vortices. The vortices were not initially in mutual equilibrium. The authors used numerical models which represent the vortices as ellipsoids and filters out non-ellipsoidal deformation. The work by Martinsen-Burrell et al. (2006) also included a study of the problem solving the full QG dynamics which includes non-ellipsoidal deformation. The authors show that vortices can deform and overlap horizontally, hence vertically align.

We revisit the problem by first determining equilibrium states (known in the literature as vortex states or V-states) for two co-rotating vortices of uniform PV. We also address their linear stability. We use two different approaches. In the first one, we model the vortices by ellipsoids and any non-ellipsoidal deformation is filtered out. We use the QG Ellipsoidal Model (ELM) introduced in Dritschel et al. (2004). ELM has already been used to determine V-states and address their linear stability in Reinaud and Dritschel (2005). In the second approach, we determine $\mathrm{V}$-states for the full QG dynamics, following previous works, see Reinaud and Dritschel (2002), Reinaud (2019).

In both cases, the equilibria found consist of tilted vortices. These vortices can nearly aligned slantwise if they are moderately offset horizontally. We show that most equilibria are stable. This is true except for narrow regions of the parameter space, in particular if the vortices are prolate. For oblate vortices, the equilibria can be unstable over a larger part of the parameter space, when the vortices are little offset horizontally.

We then study the nonlinear evolution of pairs of vortices. We either start from V-states, or from non-equilibrium, upright-standing spheroidal vortices, and we examine the ability of 
the vortices to align. Starting from a non-equilibrium state, vortices tilt and oscillate around a nearby $\mathrm{V}$-state. If the nearby $\mathrm{V}$-state is stable, the evolution is normally non-destructive, and the vortices retain (most of) their potential vorticity. On the contrary, if the nearby Vstate is unstable, vortices may shed significant amounts of filamentary potential vorticity away from the main structures. This allows the vortices to move closer together in the horizontal direction, hence to further align vertically.

The paper is organised as follows. Section 2 describes the basic equations. V-states and their linear stability are discussed in section 3 . The nonlinear evolution of the vortex pairs is presented in section 4 . Conclusions are presented in section 5 .

\section{Formulation}

We consider an inviscid, rapidly-rotating, stably-stratified fluid. We assume that the flow evolution is adiabatic. We denote $f$ the Coriolis frequency and $N$ the buoyancy frequency, both assumed constant. We denote $U$ a characteristic horizontal velocity scale, and $L$ and $H$ the horizontal and vertical length scales respectively. If $F r^{2} \ll R o \ll 1$, where $F r=U /(N H)$ is the Froude number, $R o=U /(f L)$ is the Rossby number, Euler's equations for a rotating, stratified fluid can be asymptotically expanded to give the QG equations,

$$
\begin{gathered}
q=\frac{\partial^{2} \varphi}{\partial x^{2}}+\frac{\partial^{2} \varphi}{\partial y^{2}}+\frac{\partial^{2} \varphi}{\partial z^{2}}, \\
\frac{\partial q}{\partial t}+u \frac{\partial q}{\partial x}+v \frac{\partial q}{\partial y}=0, \\
u=-\frac{\partial \varphi}{\partial y}, \quad v=\frac{\partial \varphi}{\partial x} .
\end{gathered}
$$

In the equations above, $q$ defined by (1) is the QG potential vorticity anomaly (hereinafter referred to as PV for simplicity) and $\varphi$ is the streamfunction. Equation (2) states PV is materially conserved in absence of frictional and adiabatic effects. In (1) the vertical direction $z$ corresponds to the physical vertical direction stretched by the constant factor $N / f$. Note that, in practise $N / f \gg 1$ in large parts of the oceans (see Dijkstra 2008). For uniform-PV vortices, the PV distribution, hence the flow dynamics can be fully characterised by the vortex boundaries alone. The system still retains formally an infinite number of degrees of freedom.

The problem can be simplified by modelling the vortices as ellipsoids of uniform PV and filtering out any non-ellipsoidal deformation. In that case, the ellipsoidal vortex $i$ is fully characterised by its centre $\boldsymbol{X}_{i}=\left(X_{i}, Y_{i}, Z_{i}\right)$ and a $3 \times 3$ symmetric shape matrix $\mathcal{B}_{i}=\left(\mathcal{B}_{i}\right)_{k l}$ such that the equation defining the ellipsoid surface reads $\left(\boldsymbol{x}-\boldsymbol{X}_{i}\right) \mathcal{B}_{i}^{-1}\left(\boldsymbol{x}-\boldsymbol{X}_{i}\right)^{T}=1$. Only 7 of the 12 variables are independent and time dependent for each ellipsoidal vortex. Indeed, the absence of vertical advection guaranties that $Z_{i}$ and $\left(\mathcal{B}_{i}\right)_{33}$ are time-independent, and the shape matrix $\mathcal{B}_{i}$ is symmetric by construction. The evolution of the vortex centre and its shape matrix are governed by

$$
\begin{aligned}
\frac{\mathrm{d} \boldsymbol{X}_{i}}{\mathrm{~d} t} & =\boldsymbol{u}\left(\boldsymbol{X}_{i}, t\right), \\
\frac{\mathrm{d} \mathcal{B}_{i}}{\mathrm{~d} t} & =\mathcal{S}_{i} \mathcal{B}_{i}+\mathcal{B}_{i} \mathcal{S}_{i}^{T},
\end{aligned}
$$

where the velocity $\boldsymbol{u}$ and the flow matrix $\mathcal{S}_{i}$ both derive from the Hamiltonian $H$ of the system

$$
\boldsymbol{u}\left(\boldsymbol{X}_{i}, t\right)=-\frac{1}{\kappa_{i}} \mathcal{L} \frac{\partial H}{\partial \boldsymbol{X}_{i}}, \quad \mathcal{S}_{i}=-\frac{10}{\kappa_{i}} \mathcal{L} \frac{\partial H}{\partial \mathcal{B}_{i}},
$$


where $H=E /(4 \pi)$ is the total energy of the system rescaled by $4 \pi$, with

$$
E=\frac{1}{2} \iiint|\nabla \varphi|^{2} \mathrm{~d}^{3} \boldsymbol{x} .
$$

In the equations, $\kappa_{i}$ is the potential vorticity integrated on the volume of vortex $i$ rescaled by $4 \pi$. Hence, $\kappa_{i}=(4 \pi)^{-1} \iiint_{V_{i}} q_{i} \mathrm{~d}^{3} \boldsymbol{x}=q_{i} V_{i}=a_{i} b_{i} c_{i} q_{i} / 3$ where $a_{i}, b_{i}$ and $c_{i}$ are the vortex $i$ semi-axis lengths. Finally, the matrix $\mathcal{L}$ is

$$
\mathcal{L}=\left(\begin{array}{ccc}
0 & -1 & 0 \\
1 & 0 & 0 \\
0 & 0 & 0
\end{array}\right)
$$

The Hamiltonian $H$ can be split into two parts. The first part of $H$ corresponds to the selfenergy of the ellipsoidal vortices. This part is known analytically, see Chandrasekhar (1969). The second part of $H$ corresponds to the energy of the interaction between the different ellipsoidal vortices and it can be efficiently estimated. We first determine for each ellipsoid the location and the strength of a set of singularities which induce the same streamfunction as the ellispoid, outside of the ellipsoid, within a prescribed order of accuracy in $1 / d$. Here $d$ is the distance between the centre of the ellipsoid and the evaluation point, see the details in Dritschel et al. (2004). We use here 13 singularities per vortex, and the streamfunction is accurate to $\mathcal{O}\left(d^{-9}\right)$. The interaction part of the Hamiltonian $H$ then corresponds to the interaction energy induced by the sets of singularities.

We can use this formalism to determine V-states for a pair of interacting ellipsoidal vortices. We set the vortex centres and we determine the shape matrices $\mathcal{B}_{i}$ such that $\mathcal{S}_{i} \mathcal{B}_{i}+\mathcal{B}_{i} \mathcal{S}_{i}^{T}=$ $0, i=1,2$, in a reference frame rotating at a constant angular velocity $\Omega$ while imposing the conservation of the ellipsoids volume. This is done using an iterative method, see Reinaud and Dritschel (2005). For a given V-state, the iterative procedure is stopped when a converged state is reached. Convergence is reached when the root mean squared corrections to the coefficients of the shape matrices is less than $10^{-12}$.

For a pair of ellipsoids there are 14 degrees of freedom. For the linear stability analysis, only the relative position of the vortex centres matters, reducing the number of degrees of freedom to 12. To avoid spurious modes, we only impose that the perturbation conserves, linearly, the volume of each vortex as well as the angular impulse, reducing the number of degrees of freedom to 9. Details of the approach are provided in Reinaud and Dritschel (2005).

V-states are also sought for the full QG dynamics. They are obtained by making, at all vertical levels, the contours bounding the uniform PV vortices converge to streamlines, $\varphi^{*}=$ $\varphi-(1 / 2) \Omega\left(x^{2}+y^{2}\right)=$ constant, in a reference frame steadily rotating with the equilibrium at the angular velocity $\Omega$. This is also done using an iterative method. The linear stability is determined by analysis modes of deformation of the vortex bounding contours and include modes corresponding to the displacement of the contours. Details of the approach can be found in Reinaud (2019). It is important to note that in both ELM and the full QG dynamics, the fluid domain is explicitly unbounded in all directions. The boundary conditions is that the streamfunction $\varphi$ vanishes at infinity. Therefore, the analysis does not suffer from the presence of boundary conditions at an arbitrary finite distance or the effects of periodic images of the vortices in a periodic domain.

In both approaches, instability modes have a time dependence $\propto \mathrm{e}^{\sigma t}$ where $\sigma=\sigma_{r}+\mathrm{i} \sigma_{i} \in \mathbb{C}$. Hence the real part $\sigma_{r}$ of $\sigma$ is the mode's growth rate while $\sigma_{i}$ is its frequency.

To investigate the nonlinear evolution in the full QG regime, simulations are performed the unbounded QG contour surgery algorithm. The method takes its origin in the Contour Dynamics method introduced by Zabusky et al. (1979) for two-dimensional flows. A procedure of 'surgery' was introduced by Dritschel (1988a) and Dritschel (1989a) to control the complexity of the contours representing the vortex boundaries. The two-dimensional method was 


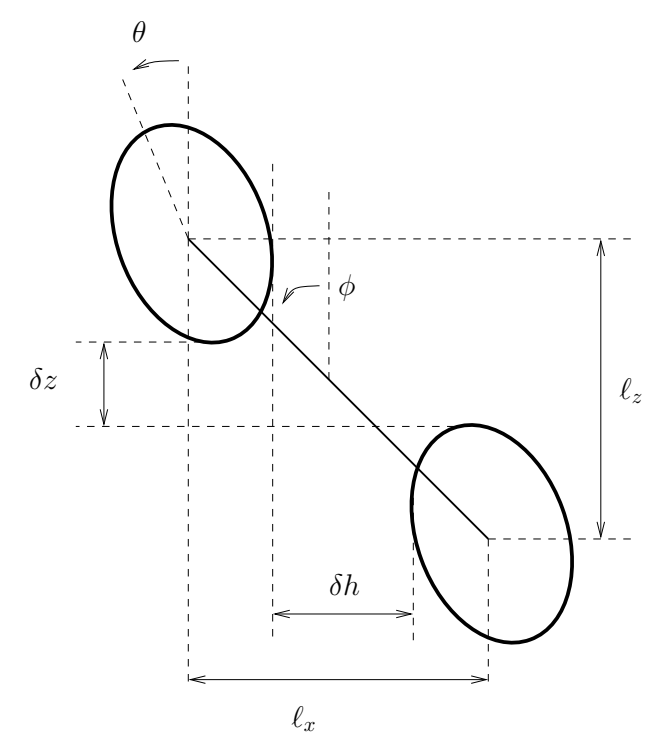

Figure 1. General geometry and definition of the parameters

extended to three-dimensional QG equations by Dritschel and Saravanan (1994), Dritschel (2002). The method are purely Lagrangian.

\section{V-states and their linear stability}

\subsection{Ellipsoidal $V$-states}

We first determine ellipsoidal V-states. To reduce the size of the parameter space, we restrict attention to equilibria for pairs of vortices having the same volume, the same height-to-width aspect ratio $h / r$ and opposite PV $q_{1}=-q_{2}$. Here, $h$ is the half height of the vortices and $r$ is their mean horizontal radius. Without loss of generality we set $q_{1}=2 \pi$. The geometry of the configuration is presented in figure 1. V-states are organised in families of equilibria. V-states within a family have the same prescribed height-to-width aspect ratio $h / r$, and the same prescribed minimum distance $\delta z$ separating the vortices in the vertical direction. The states differ by the horizontal distance between the vortices. Without loss of generality, we set $Y_{1}=Y_{2}=0$, and the vortices are separated in the $x$-direction. The centres of the vortices are separated in the horizontal direction by $\ell_{x}=X_{1}-X_{2} \geq 0$ and in the vertical direction by $\ell_{z}=Z_{1}-Z_{2}<0$, without loss of generality. It should be noted that $\left|\ell_{z}\right|=\delta z+2 h$. The length scale of the problem is set by imposing that the full height of the vortex pair $\delta z+4 h=1$.

We define two angles to characterise the slantwise alignment of the vortices. The angle $\phi=\arctan \left(\left|\ell_{x} / \ell_{z}\right|\right)$ is the angle between the vertical direction and the line passing through the vortex centres is referred to as the vortex inter-centre angle. The angle $\theta$ is the minimum angle between the vertical direction and one of the three semi-axes of the ellipsoid and is referred to as the vortex tilt angle. The direction of the ellipsoid semi-axes are given by the eigenvectors of the shape matrices $\mathcal{B}_{i}$.

In this part, we restrict attention to $\mathrm{V}$-states with $\delta z=0$, where the vortices occupy regions of the fluid domain contiguous in the vertical direction. For each prescribed $h / r$, we start by determining a first $\mathrm{V}$-state where the vortices are well separated in the horizontal direction, namely $\ell_{x} / r=8$. The first guess for the equilibrium consist of two upright-standing spheroids. It should be noted an upright-standing spheroid alone is a steady state. We then use our iterative method to correct the shape of the two vortices until we reach an equilibrium. We then decrease the distance separating the vortex centres by a small amount, $\Delta \ell_{x}=10^{-3} r$, and 

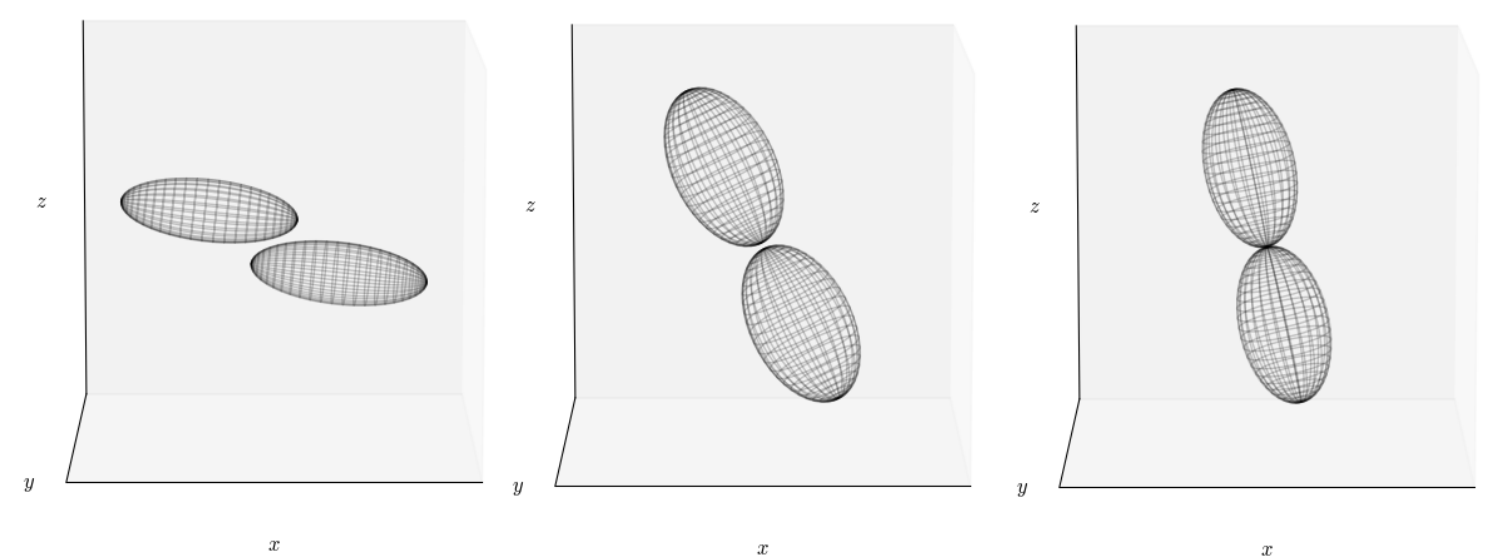

Figure 2. View on the pair of ellipsoidal vortices in mutual equilibrium at $\delta z=0$ for $h / r=0.5$ and $\ell_{x} / r=2$ viewed in the domain $[-1.5,1.5]^{3}$ (left); $h / r=1.5$ and $\ell_{x} / r=1.46$ viewed in the domain $[-0.6,0.6]^{3}$ (centre); $h / r=4$ and $\ell_{x} / r=1.2$ viewed in the domain $[-0.6,0.6]^{3}$ (right).
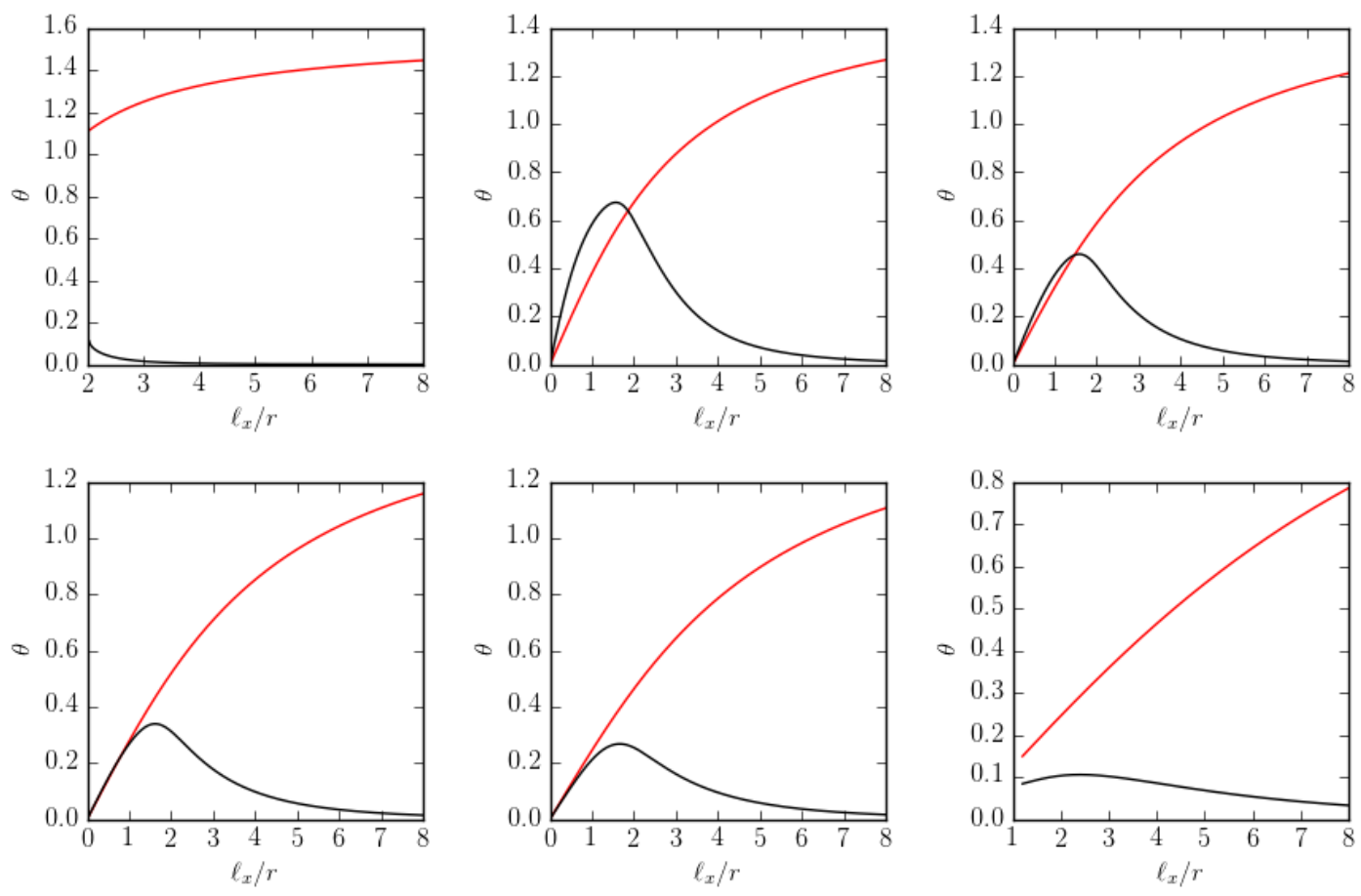

Figure 3. Equilibrium ellipsoidal vortex tilt angle $\theta$ (black) and inter-centre angle $\phi$ from the vertical (red) vs the centre normalised horizontal separation $\ell_{x} / r$ for $h / r=0.5,1.25,1.5,1.75,2$ and 4 (colour online).

resume the iterative method to find a new equilibrium. The procedure is continued until the two vortices are perfectly aligned vertically, $\ell_{x}=0$ or until no steady state can be found. Recall that the length scale of the problem is set, without loss of generality, by setting $4 h+\delta z=1$ such that $h=0.25$ for $\delta z=0$.

Figure 2 shows the shape of ellipsoidal equilibrium vortices for three examples with $h / r=$ $0.5,1.5$ and 4 respectively. The first two V-states shown for $h / r=0.5$ and $\ell_{x} / r=2$, and $h / r=1.5$ and $\ell_{x} / r=1.46$ are linearly unstable while the last $\mathrm{V}$-state with $h / r=4$ and $\ell_{x} / r=1.2$ is linearly stable. The vortices of the $\mathrm{V}$-state with $h / r=0.5$ and $\ell_{x} / r=2$ are strongly deformed and can be seen to overlap horizontally even if $\ell_{x} / r=2$.

We consider two notions of alignment for the vortices. We first consider the slantwise align- 

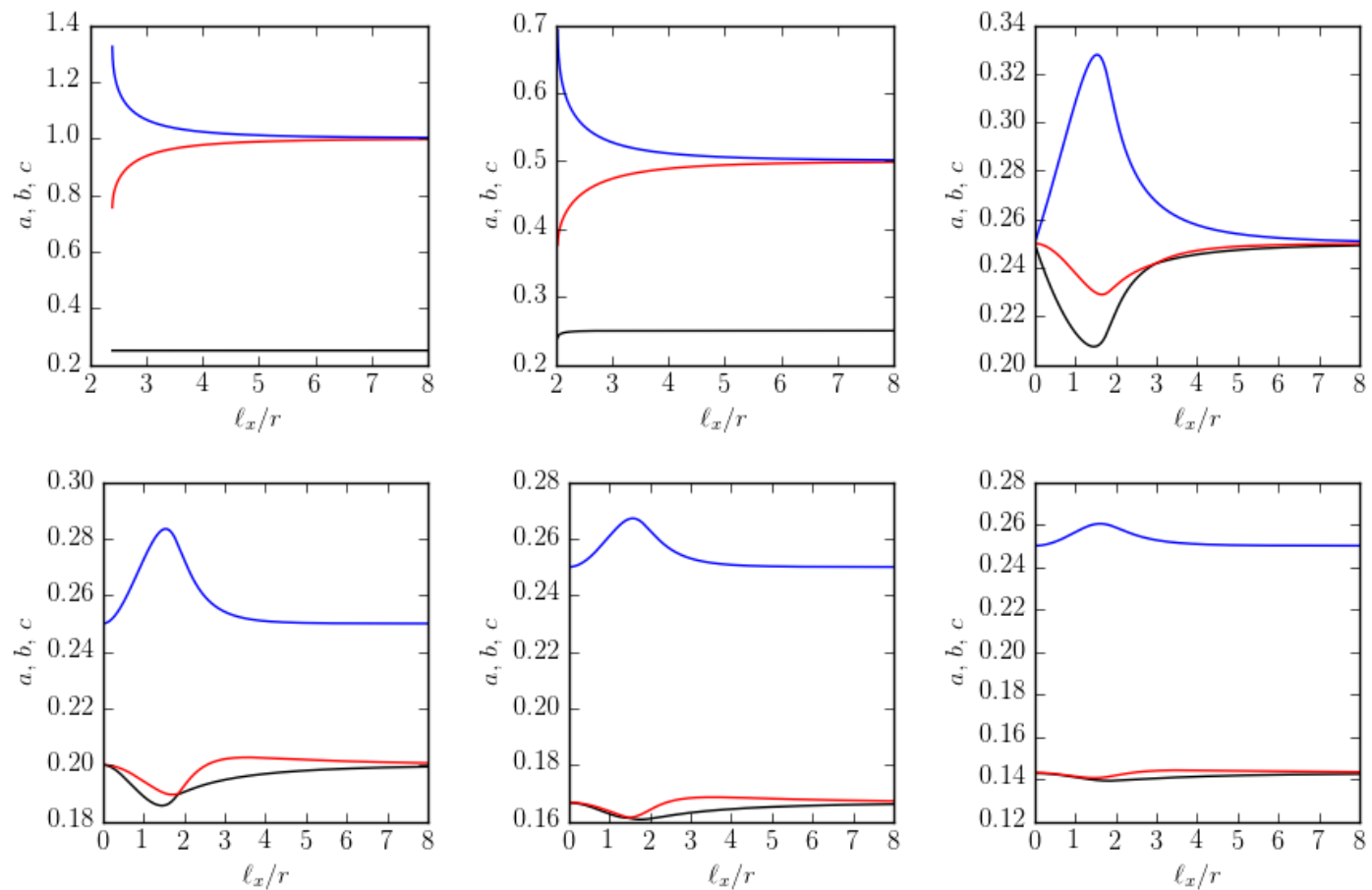

Figure 4. Semi-axis lengths $(a, b, c)$ for the ellipsoidal equilibrium vortices vs the centre normalised horizontal separation $\ell_{x} / r$ for $h / r=0.25,0.5,1.0,1.25,1.5$ and $1.75(\delta z=0)$. The length scale is set by imposing that the total height of the configuration $4 h=1 . a$ is shown in black, $b$ in red and $c$ in blue (colour online).

ment of the vortices. Vortices are said to align slantwise if the vortices tilt such that one of their semi-axis nearly aligns with the axis joining the vortex centres, in other words if $\phi \simeq \theta$. We also consider the vertical alignment of the vortices where horizontal cross-sections of the two vortices overlap, despite being in different horizontal planes. Recall that the vortices centres are aligned along the $x$-axis. Overlapping occurs when the distance between the two innermost edges of the vortices changes sign, namely when $\ell_{x}-\sqrt{\left(\mathcal{B}_{1}\right)_{11}}-\sqrt{\left(\mathcal{B}_{2}\right)_{11}}<0$, since $\sqrt{\left(\mathcal{B}_{i}\right)_{11}}$ is the distance between the centre of vortex $i$ and its edges in the $x$-direction.

Figure 3 shows the vortex tilt angle $\theta$ and the vortex centre angle $\phi$ as a function of the normalised horizontal separation $\ell_{x} / r$. First, $\phi \rightarrow \pi / 2$ as $\left|\ell_{x} / \ell_{z}\right| \rightarrow \infty$, by definition of $\phi$. Then, for a family of $\mathrm{V}$-state with a prescribed (finite) $\ell_{z}, \phi$ decreases monotonically as $\ell_{x}$ is decreased. By definition, $\phi \rightarrow 0$ as $\ell_{x} \rightarrow 0$. On the other hand, for $\left|\ell_{x}\right| \gg\left|\ell_{z}\right|$, the vortices are standing nearly upright such that $\theta \rightarrow 0$. As $\ell_{x}$ is decreased the vortices first increases their tilt angle until it reaches a maximum for some $\ell_{x}^{s}$. Then it decreases since the vortices tend to stand upright when both $\ell_{x} \rightarrow 0$ and $\ell_{x} \rightarrow \infty$. For prolate vortices, as shown in the example $h / r=0.5$ in figure 3 , we were not able to find $\mathrm{V}$-states for $\ell_{x} / r<2$. The numerical method diverges. We will see that this is related to a sharp increase of the vortex deformation. In this case, and in the range where $\mathrm{V}$-states are obtained, the vortices are too far apart to align slantwise. On the other hand, for $h / r=1.25,1.5,1.75$ there is a range of $\ell_{x} / r$ for which $\phi \simeq \theta$. The range roughly correspond to $\left[0, \ell_{x}^{s}\right]$, with $\ell_{x}^{s} / r \simeq 1.5$.

We next characterise the shape of vortices of the $\mathrm{V}$-states by calculating the semi-axis lengths $a_{i}, b_{i}$ and $c_{i}$ for the vortices. The semi-axis lengths correspond to the square root of the eigenvalues of the shape matrices $\mathcal{B}_{i}$. Again, by symmetry the two ellipsoidal vortices have the same semi-axis lengths, hence the values for only one vortex are discussed and are simply referred to as $(a, b, c) \equiv\left(\alpha_{j}\right)_{j=1,2,3}$. Results are presented in figure 4 for $h / r=$ $0.25,0.5,1,1.25,1.5$ and 1.75 .

For $h / r=0.25$ and $h / r=0.5$, two values of $\partial \alpha_{j} / \partial \ell_{x} \rightarrow \pm \infty$, for some finite $\ell_{x}^{e}$. At this point, 
the vortices would need to deform infinitely to steadily withstand the strain they induce onto each other. In other words, there are no $\mathrm{V}$-state along this branch for a horizontal separation less than $\ell_{x}^{e}$. The iterative procedure fails to find steady states for $\ell_{x}<\ell_{x}^{e}$. A few states (on a different branch) could be found well beyond the point of divergence of the derivatives $\partial \alpha_{j} / \partial \ell_{x}$, i.e. for $\ell_{x} \ll \ell_{x}^{e}$, but we could not reconstruct a full branch for $0<\ell_{x}<\ell_{x}^{e}$.

For the other values of $h / r$ investigated $(h / r \geq 1), \partial \alpha_{j} / \partial \ell_{x}$ remains finite for all $\ell_{x}$ and the full branch of $\mathrm{V}$-states can be determined. We also note that the relative maximum deformation of the vortices decreases as $h / r$ is increased. This is due to the fact that by increasing the vortex heights, their centres are further apart and overall the intensity of the strain they induce onto each other decreases.

For $\ell_{x} / r \gg 1$, two semi-axis lengths remain nearly equal, indicating that the vortices remain close to spheroids. This also corresponds to regions of the parameter space where $\theta$ is small, hence the vortices are also nearly standing upright. Then the vortices deform for moderate values of $\ell_{x} / r$. This also corresponds to the range of $\ell_{x} / r$ for which the vortices tilt the most.

Figure 5 show the angular impulse

$$
J=\frac{1}{2} \iiint q\left(x^{2}+y^{2}\right) \mathrm{d}^{3} \boldsymbol{x},
$$

the total energy $E$, and the angular velocity $\Omega$ for the ellipsoidal $\mathrm{V}$-states as functions of $\ell_{x} / r$ for $h / r=0.25,1.25,1.5$, and 1.75. Both $J$ and $E$ are motion invariants. Generally, the angular impulse decreases as $\ell_{x}$ is decreased, and this trends is simply due to the overall dominant contribution of the horizontal separation between the vortex centres to $J$. The angular velocity $\Omega$ increases as $\ell_{x}$ is decreased due to the increase of the velocity that the vortices induce on each other as they get closer. On the other hand, the trend for the total energy $E$ of the ellipsoidal V-states is not monotonic as $\ell_{x}$ is decreased. First, for $\ell_{x} \gg 1$, as the vortices get closer, their interaction becomes stronger and the interaction energy increases. On the other hand, as seen in figure 4 , there is a range of $\ell_{x}$ for which the vortices strongly deform. The more the vortices deform the lower their self-energy is, for the prescribed vortex PV and volume. The decrease in self-energy can be larger than the increase of interaction energy as $\ell_{x}$ is decreased. As a consequence, they can be a range where the total energy overall decreases as $\ell_{x}$ is decreased. For lower values of $\ell_{x}$, the vortices are less deformed and the self-energy increases again.

We next present the results of the linear stability analysis. Figure 6 shows the maximum growth rate of instability as a function of the normalised horizontal centre separation $\ell_{x} / r$ for $\delta z=0$ and $h / r=0.25,0.5,1.25,1.5,1.75$ and 4 . For $h / r=0.25$ and $h / r=0.5$, an unstable mode emerges for $\ell_{x}^{c} / r=2.4 \simeq \ell_{x}^{e} / r$ and $\ell_{x}^{c} / r=2.05 \simeq \ell_{x}^{e} / r$, respectively. As mentioned above, we could not continue the branch of solutions for $\ell_{x} / r<\ell_{x}^{e} / r$. Recall that $\ell_{x} / r \rightarrow \ell_{x}^{e}$ corresponds to $\partial \alpha_{j} / \partial \ell_{x} \rightarrow \infty$. For $h / r=1.25,1.5$ and 1.75 we have obtained the full branch of $\mathrm{V}$-states in the range $\ell_{x} / r \in[0,8]$. Figure 6 shows that the most of the $\mathrm{V}$-states are stable, except for a very narrow range of values for $\ell_{x} / r$. For these values of $h / r, \partial \alpha_{j} / \partial \ell_{x}$ remains bounded for all $\ell_{x}$. The critical values of $\ell_{x} / r$ where instability is found decreases monotonically as $h / r$ is increased. For larger values of the height-to-width aspect, $h / r=2$ and 4 , no instability was found. It should also be noted that we could not find ellipsoidal unstable modes for $h / r \in[0.65,1.1]$ even if one would expect one. It is unclear why it is the case. It could either due the fact that the width of the range where the instability tends to 0 , or to the fact that the projection of the instability modes onto ellipsoidal deformation modes is vanishing. As we will see in the next part, there are indeed unstable mode in the full QG regime for this range of values for $h / r$.

Figure 7 shows the evolution of ellipsoidal vortices semi-axis lengths for two examples of unstable ellipsoidal V-states. In both cases, the simulation are run with ELM. Recall that in this model, the vortices remains ellipsoidal bodies at all time, and any non-ellipsoidal deformation is filtered out. The first case considered is for $h / r=0.25$ and $\ell_{x} / r=2.4$, the 

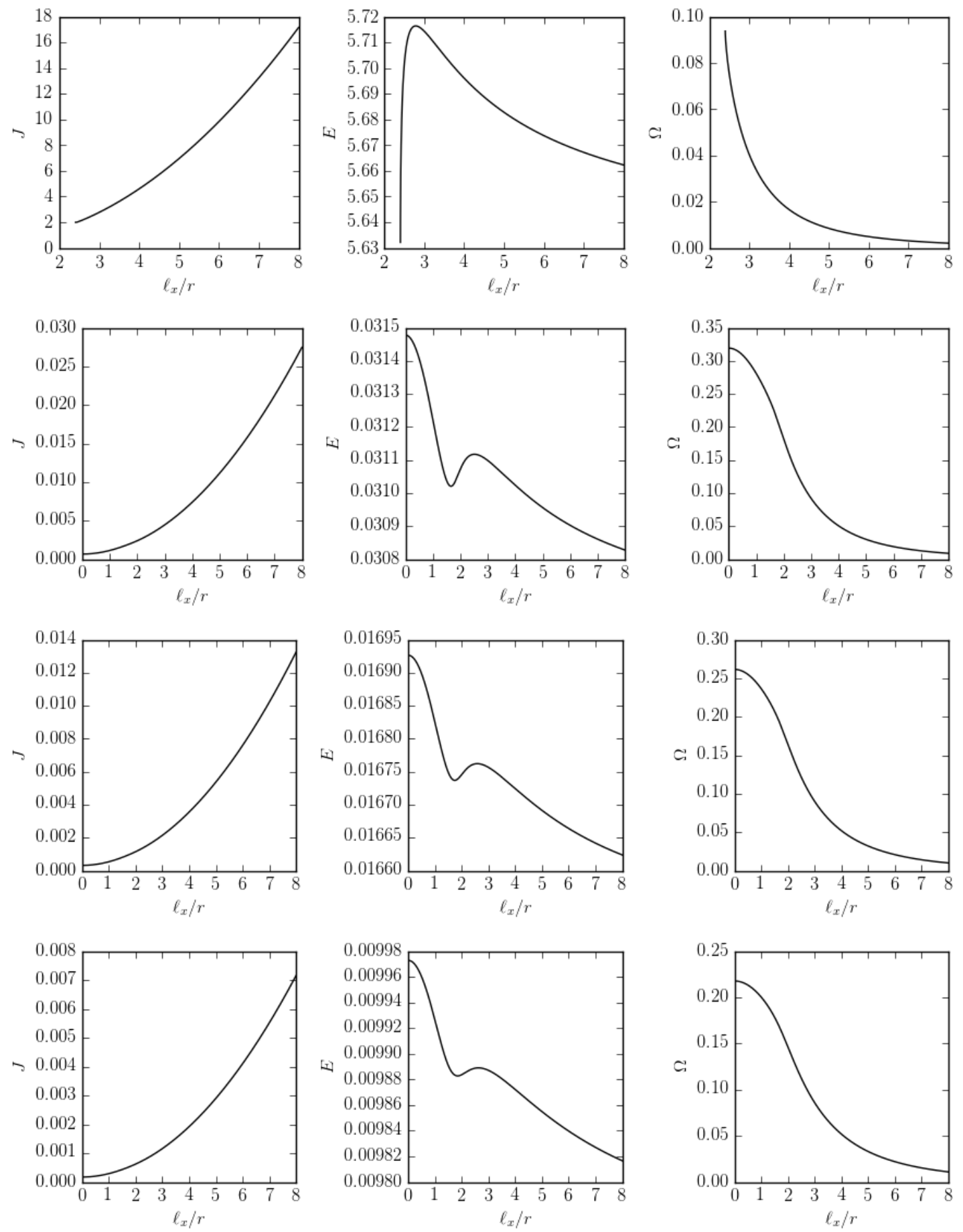

Figure 5. Angular impulse $J$, total energy $E$ and rotation velocity $\Omega$ vs the centre normalised horizontal separation $\ell_{x} / r$ for ellipsoidal equilibrium vortices with $h / r=0.25$ (first row), 1.25 (second row), 1.5 (third row) and 1.75 (fourth row).

ellipsoidal V-state is strongly deformed with $a \simeq 0.25, b \simeq 0.756$ and $c \simeq 1.325(a / \sqrt{b c} \simeq h / r$ - the equality would be exact if the vortices were upright-standing). We do not introduce any initial perturbation, and perturbations grow from numerical noise. As seen in figure 7 , the vortices initially do not deform as they are in mutual equilibrium until $t \simeq 155$. Then, we observe quasi-periodic oscillations for the ellipsoidal vortices semi-axis lengths. The two 

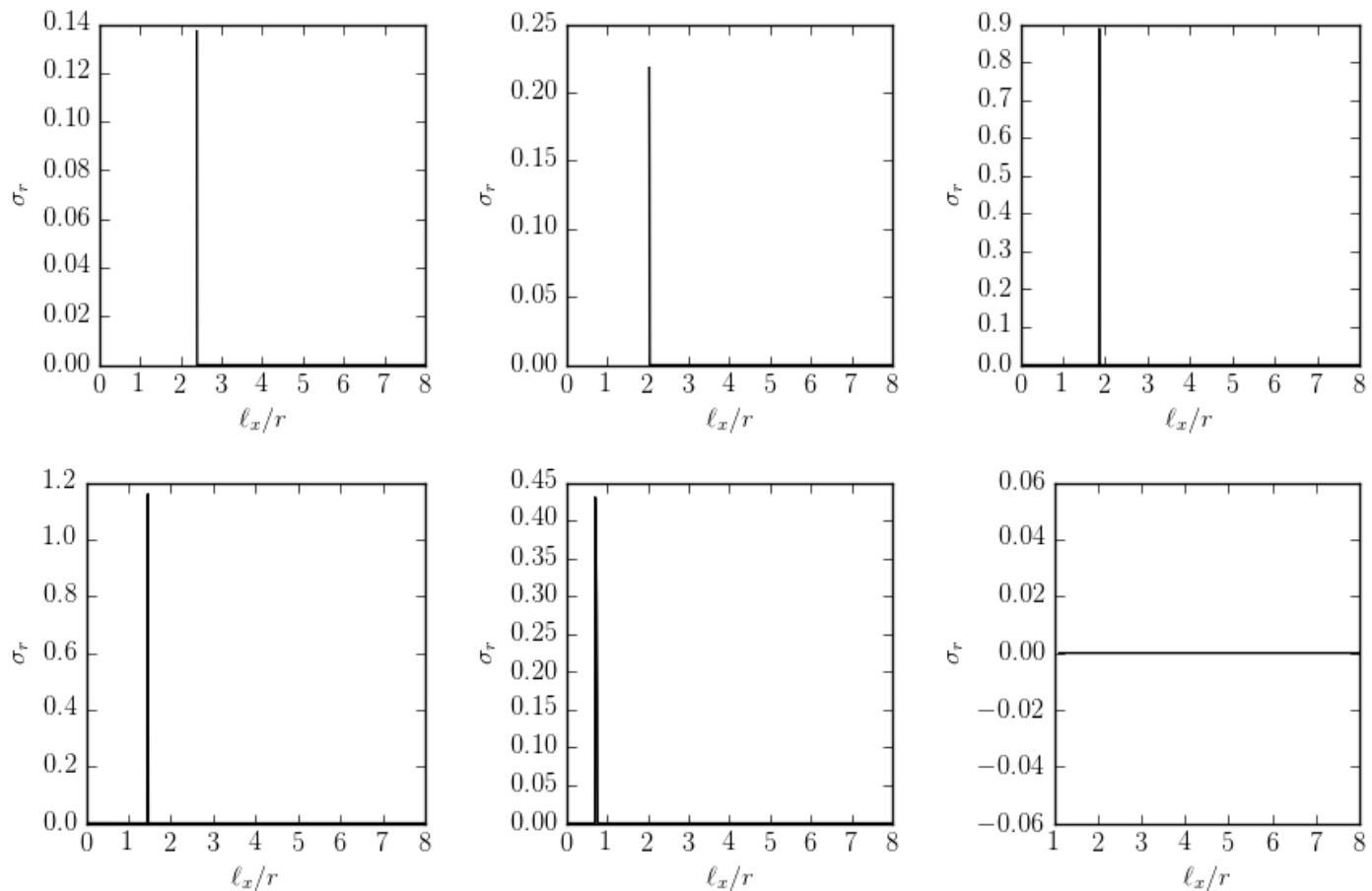

Figure 6. Growth rate $\sigma_{r}$ of most amplified ellipsoidal instability mode for ellipsoidal equilibrium vortices vs the horizontal normalised centre separation $\ell_{x} / r$ at $\delta z=0$ and $h=0.25$. for from left to right, then top to bottom $h / r=0.25,0.5,1.25,1.5,1.75$ and 4 .

vortices oscillate in a similar way until $t \simeq 380$, when the symmetry breaks and vortex 2 becomes much more deformed than vortex 1 . The relative oscillations have a large amplitude (the semi-axis lengths can double or even triple). A qualitatively similar evolution is obtained for the unstable $h / r=0.5$ case (not shown). For the second case, $h / r=1.5$, and $\ell_{x} / r=$ 1.46. The vortices remain at equilibrium until $t \simeq 10$, then the semi-axis lengths start to oscillate. In this case, the amplitude of the oscillations are very small, with a maximum of $\alpha_{j}-\alpha_{j}^{0} \simeq 6 \times 10^{-4} \alpha_{j}^{0}$. In other words, the vortices remain almost unchanged during their nonlinear evolution. Similar results are obtained for the unstable V-states for $h / r=1.25$ and 1.75 (results not shown). This illustrates, on specific examples, a generic trend. Unstable V-states of oblate vortices can strongly deform while unstable V-states of prolate vortices are very little affected by the instability in the nonlinear regime.

\subsection{Full $Q G \quad V$-states}

We next turn our attention to V-states under the full QG dynamics. Each vortices is mapped by 61 horizontal layers. Two symmetries are imposed to reduce the calculation cost. First, the vortices centres are aligned along the $x$-direction, and each vortex is symmetric with respect to the $x z$-plane. Second, vortex 2 is the image of vortex 1 by the central symmetry with respect to the origin. We also determine the ellipsoids which best fits the vortices of the V-state. The best-fitted ellipsoid is the ellipsoid of same centre

$$
\boldsymbol{X}^{e}=\iiint_{\text {vortex }} \boldsymbol{x}^{\prime} \mathrm{d}^{3} \boldsymbol{x}^{\prime} / \iiint_{\text {vortex }} \mathrm{d}^{3} \boldsymbol{x}^{\prime}
$$

and sharing the same second order geometrical moments

$$
I_{i j}=\iiint_{\text {vortex }}\left(x_{i}-X_{i}^{e}\right)\left(x_{j}-X_{j}^{e}\right) \mathrm{d}^{3} \boldsymbol{x}^{\prime}
$$



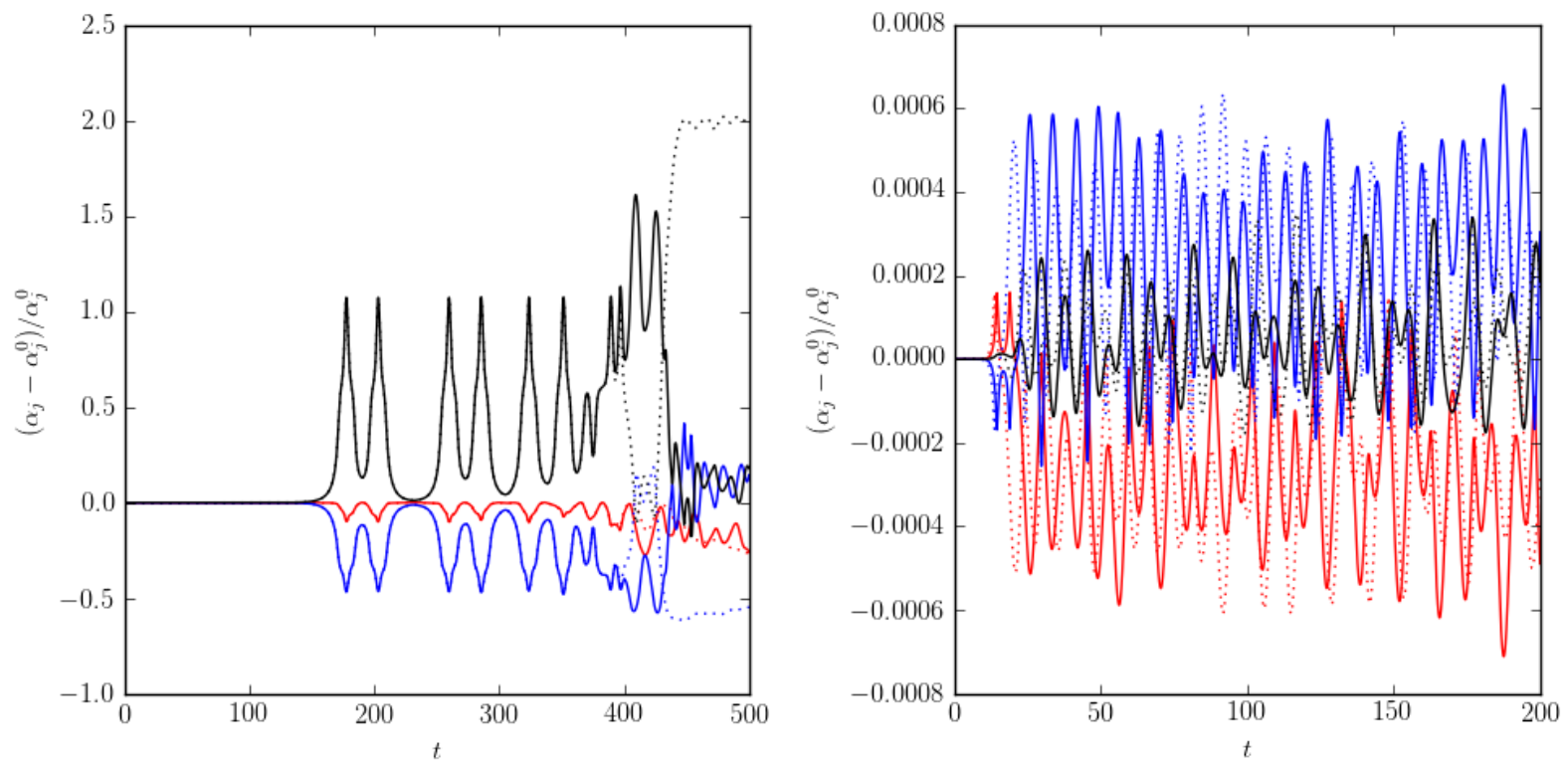

Figure 7. Evolution (using the dynamical ellipsoidal model) of the ellipsoid semi-axis lengths relative oscillations for unstable ellipsoidal equilibrium vortices with $h / r=0.25 \ell_{x} / r=2.4$ (left) and $h / r=1.5, \ell_{x} / r=1.46$ (right). $\left(\alpha_{j}\right)_{j=1,2,3} \equiv(a, b, c)$ and $\alpha_{j}^{0}=\alpha_{j}(t=0)$ for vortex 1 (solid lines) and vortex 2 (dashed lines). The convention is $a \leq b \leq c$. The red curve corresponds to $a \equiv \alpha_{1}$, the blue curve to $b \equiv \alpha_{2}$ and the black curve to $c \equiv \alpha_{3}$ (colour online).

where $\left(x_{i}\right)_{i=1,2,3}=(x, y, z)$. Note that the second order moments are directly linked to the shape matrix $\mathcal{B}$ as $\mathcal{B}_{i j}=5 I_{i j} / \iiint_{\text {vortex }} \mathrm{d}^{3} \boldsymbol{x}^{\prime}$. Figure 8 shows the best-fitted ellipsoid semi-axis lengths for one vortex of the $\mathrm{V}$-states as a function of the normalised horizontal separation $\ell_{x}$ for $\delta z=0$ and for $h / r=0.25,0.5,1.0,1.5$ and 2 . Only vortex 1 is presented as the vortices are symmetric. As before, the length scale of the problem is set by imposing that the total height of the vortex pair $4 h+\delta z=1$.

For $h / r=0.25$, we observe that the rate of change of the semi-axis lengths with respect to the horizontal direction $\ell_{x}$ diverges for some finite value of $\ell_{x}$ as we already observed for the ellipsoidal V-states. Figure 9 shows the last V-state obtained in this branch. Recall, that the horizontal cross-sections of the vortex boundary of the V-states coincide with streamlines in the reference frame steadily rotating with the vortex pair. Each vortex shows a sharp inner edge, indicating the presence of stagnation points. There is no $\mathrm{V}$-state along this branch for smaller $\ell_{x}$. There should still exist at least one (different) branch of V-states for $\ell_{x}$ smaller stemming from the radially symmetric case at $\ell_{x}=0$. Indeed, any radially symmetric distribution of PV is a steady state in QG. We were not able to obtain this branch numerically (except for the trivial case $\ell_{x}=0$ ). Note that this branch may not have the symmetries that we impose.

For $h / r>0.25$, we investigate the branches of V-states by $(i)$ starting from two horizontally well-separated, $\ell_{x} / r \gg 1$, upright-standing vortices and follow the branch by reducing $\ell_{x}$, and (ii) starting from two vertically-aligned, $\ell_{x}=0$, upright-standing vortices and follow the branch by increasing $\ell_{x}$. We were able to construct (almost) full branches of $\mathrm{V}$-states. For $h / r=0.5$, it is clear from figure 8 that the semi-axis lengths of the best-fitted ellipsoids varies continuously along the branch parametrised by $\ell_{x} / r$. Only a very section of the full branch could not be determined and corresponds to a region where the slope of $\alpha_{i}=f\left(\ell_{x} / r\right)$ is very steep. For $h / r>0.5$, the curves of $a, b, c$ as functions of $\ell_{x} / r$ are in qualitative agreement with the ones obtained for the ellipsoidal V-states. In both cases, $a, b, c$ tend to the expected values for an upstanding spheroid of the prescribed height-to-width aspect ratio $h / r$ when both $\ell_{x} / r \rightarrow \infty$ and 0 . The maximum deformation is obtained for intermediate values of $\ell_{x} / r$. There is however a difference between the ellipsoidal V-states and the full 

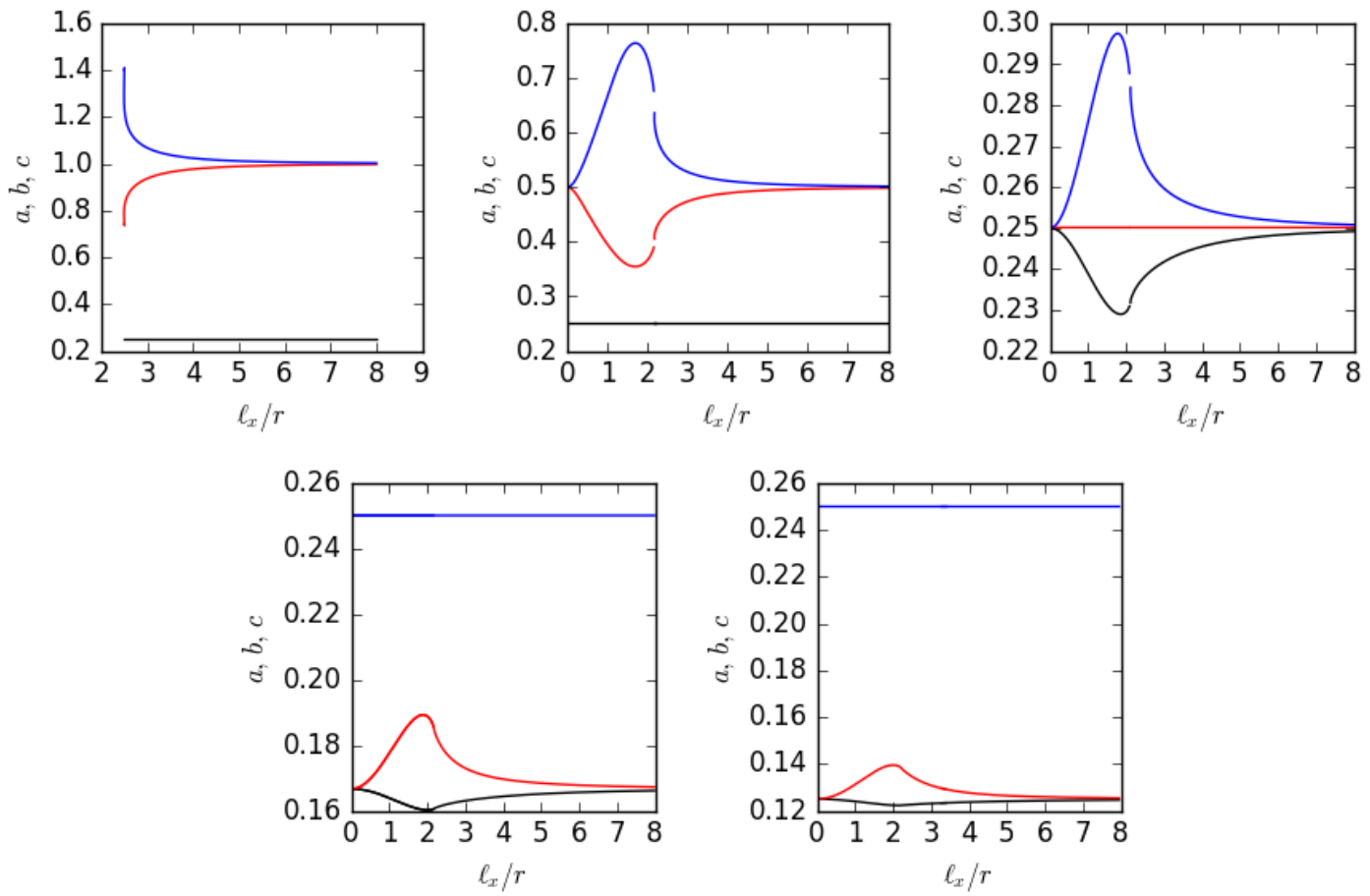

Figure 8. Best-fitted ellipsoid semi-axis lengths for the full QG equilibria vortices vs the centre normalised horizontal separation $\ell_{x} / r$ for $h / r=0.25,0.5,1.0,1.5$ and 2 and $\delta z=0$. The length scale is set by imposing that the total height of the configuration $4 h=1$. The notations $a, b, c$ and the colours are the same as in figure 7 (colour online).
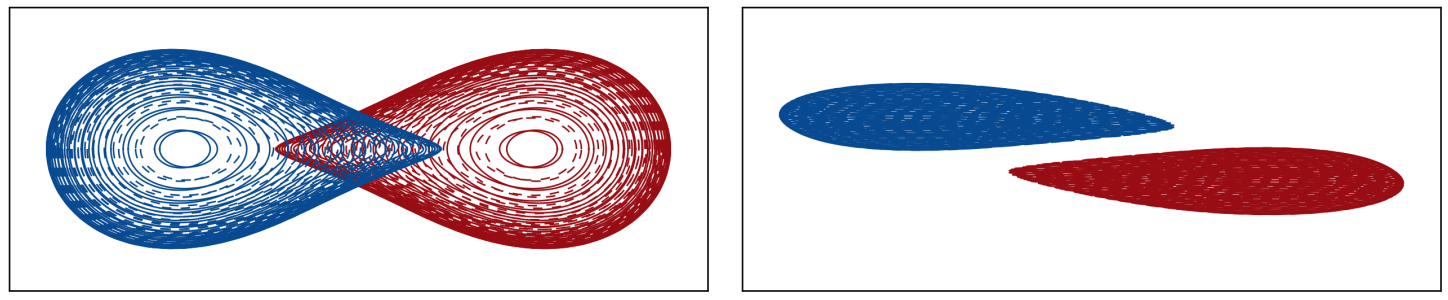

Figure 9. View on the vortex bounding contours for the end of the V-state branch for $h / r=0.25$ : Top view (left) and orthographic view at an angle of $85^{\circ}$ from the vertical (right).

QG V-states. This difference is fundamentally related to the smaller number of degrees of freedom of the ellipsoidal $\mathrm{V}$-states. This impacts the way a vortex can adapt to vertical shear. This is in particular visible when comparing the curves for $h / r=1.5$. When submitted to a vertical shear, an ellipsoidal vortex can only tilt. There is ' rigidity' which forbids high wavenumber vertical modes to deform the vortex in the vertical direction as the vortex must remain ellipsoidal. On the other hand, in the full QG model, the vortex can deform following as many vertical modes as allowed by the vertical discretisation (i.e. by the number of layers spanning the vortex). Hence a full QG V-state may deform locally, where the vertical shear is high, without having to overall tilt significantly. This means that the full QG vortices of the V-states are significantly less (overall) titled than their ellipsoidal counterparts. For the full QG V-states, the vortices do not tilt significantly and $c \simeq h$ for all $\ell_{x}$ (as seen from the fourth panel of figure 8). This is not true for the ellipsoidal V-state. Since the total height of the vortex pair is conserved by the absence of vertical advection, the titling of the vortex must be accompanied by as increase of the length of the most vertical semi-axis, see the fifth panel of figure 4. This difference has also an impact on the two other semi-axis lengths by conservation of volume $V=4 \pi a b c / 3=$ constant. Nonetheless, the maximum deformation occurs roughly 

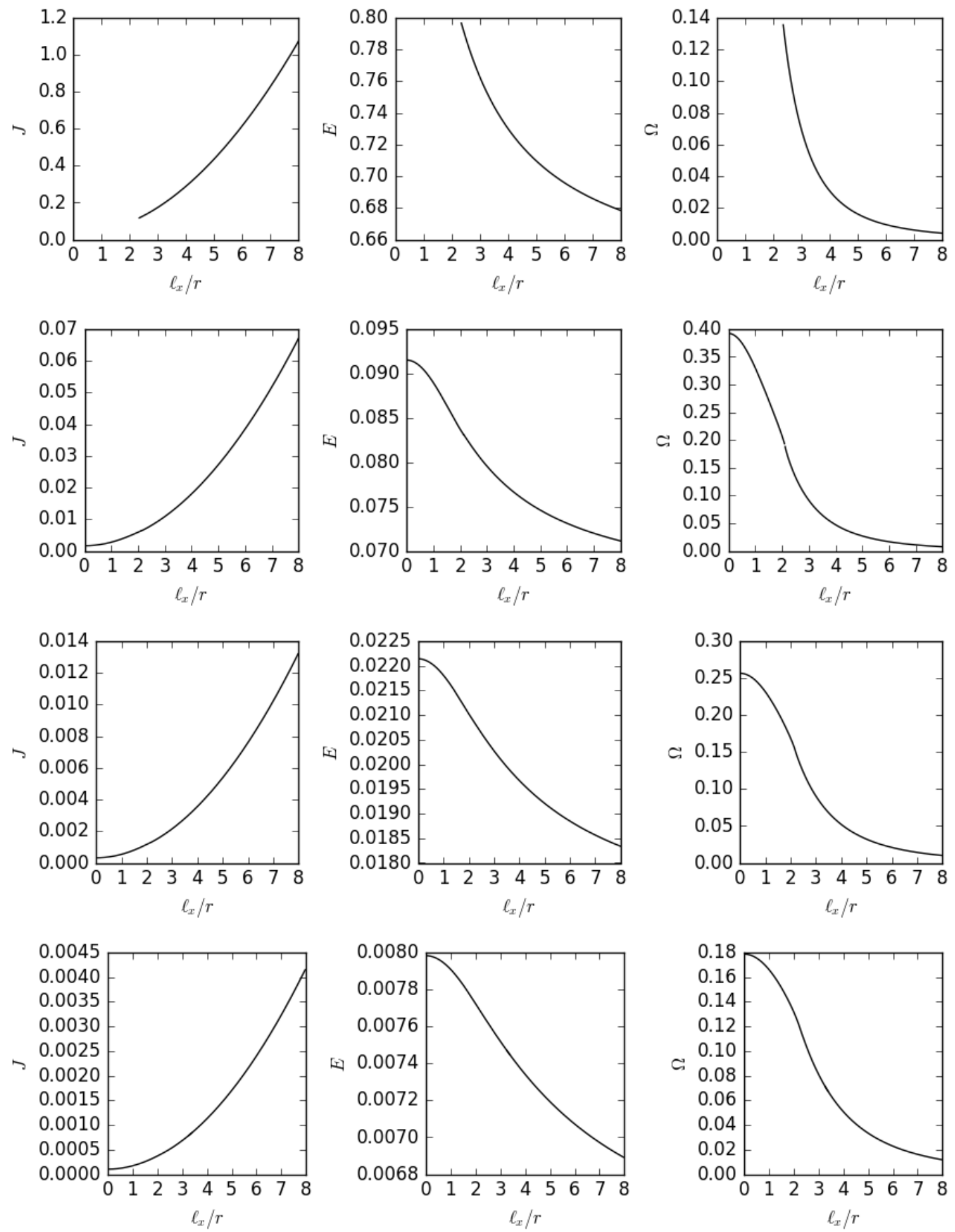

Figure 10. Angular impulse $J$, total energy $E$ and rotation velocity $\Omega$ vs the centre normalised horizontal separation $\ell_{x} / r$ for full QG equilibrium vortices with $h / r=0.5$ (first row), 1 (second row), 1.5 (third row) and 2 (fourth row).

in the same range of values for $\ell_{x} / r$, and results are overall in agreement.

Figure 10 shows the angular impulse $J$, the total energy $E$, and the angular velocity $\Omega$ as functions of $\ell_{x} / r$ for $h / r=0.5,1.5,1.5$, and 2 , again for $\delta z=0$. The trends are similar as the ones observed for the ellipsoidal V-states apart for the total energy for moderate heightto-width ratios, see the cases $h / r=1.5$. The total energy $E$ remains a monotonic function of the normalised horizontal separation $\ell_{x} / r$ in contrast with the total energy of the ellipsoidal 

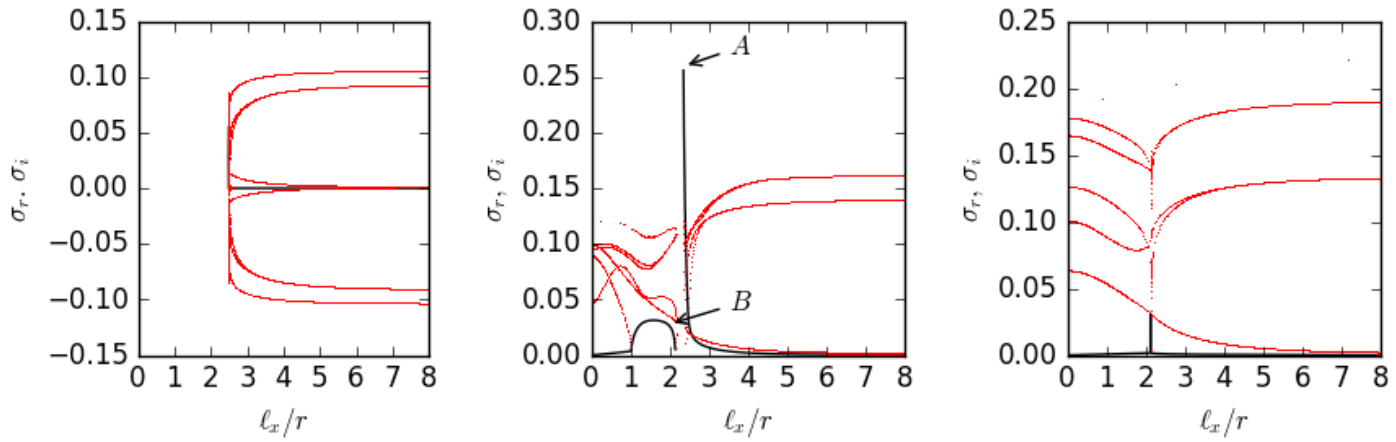

Figure 11. Growth rate $\sigma_{r}$ of most amplified instability mode for full QG two-vortex equilibria at $\delta z=0$ vs the horizontal normalised centre separation $\ell_{x} / r$ (black) and the five smallest non-zero frequencies $\sigma_{i}$ (red) for from left to right $h / r=0.25,0.5,1$ (colour online).
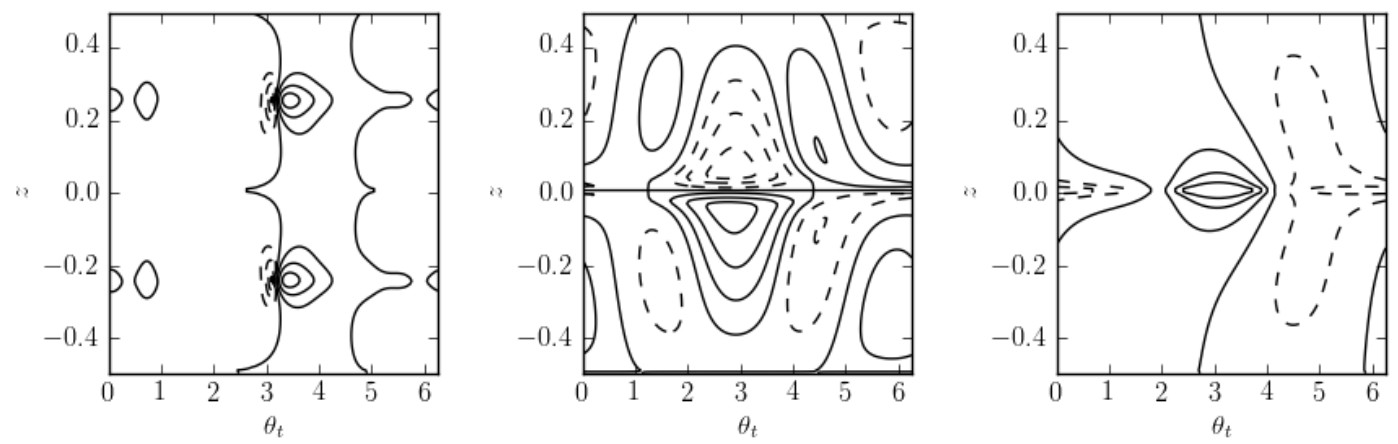

Figure 12. Spatial structure of the most amplified mode in the plane $\left(\theta_{t}, z\right)$ where $\theta_{t}$ is the travel coordinate for $h / r=0.5$ and $\ell_{x} / r=2.35$ (left), $h / r=0.5$ and $\ell_{x} / r=2$ (middle) and $h / r=1$ and $\ell_{x} / r=2.1$ (right). The solid lines (resp. dashed lines) correspond to positive, outward (resp. negative inward) deformations.

V-states. This is due to the difference in deformation. Recall that the vortex deformation has an influence on the total energy, in particular on the self-energy.

The maximum growth rate $\sigma_{r}$ of the instability modes is presented in figure 11 for $h / r=$ $0.25, h / r=0.1$ and $h / r=1$ and $\delta z=0$. For $h / r=0.25$, we observed a mode emerging very close to the end of the branch of $\mathrm{V}$-states for the minimum value of $\ell_{x} / r$. As seen before, this is associated with the formation of a 'corner' state, where the innermost edges of the vortices form a corner, indicating a stagnation point. This is very similar to what is observed for vortices sharing horizontal levels and the mode of instability associated with vortex merger, see Reinaud and Dritschel (2002). The difference is that the corners do not form at the same vertical levels. As mentioned before, we were not able to find a full branch of $\mathrm{V}$-states for small $\ell_{x}$. For $h / r=0.5$, we were able to find a full branch of solution, except a in narrow gap around $\ell_{x} / r \sim 2.1$. Decreasing $\ell_{x} / r$ from large values, we observe a first mode of instability which is again, very localised in a narrow range of $\ell_{x}$. For smaller values of $\ell_{x} / r$, there is a second mode of instability for $\ell_{x} / r \in[1,2.1]$, hence oblate $\mathrm{V}$-states are also unstable for moderate horizontal separation between the vortices. Such instabilities are not present for $h / r=1$, and the $\mathrm{V}$-states are stable except in a very narrow range around $\ell_{x} / r \simeq 2.1$.

Results for other aspect ratios $1 \leq h / r \leq 2$ also indicate the presence of an instability in a very narrow range of $\ell_{x} / r$, but no secondary instability for small $\ell_{x} / r$. Figure 11 also indicates the five smallest, non-zero, frequencies $\sigma_{i}$. It is shown that one mode with zero growth rate has a frequency which decreases faster than the others with decreasing $\ell_{x}$ to collapse to zero at the onset of instability. In other words, $\sigma$ goes from pure imaginary to real. The same exchange is observed for modes associated with vortex merger, see Reinaud and Dritschel 

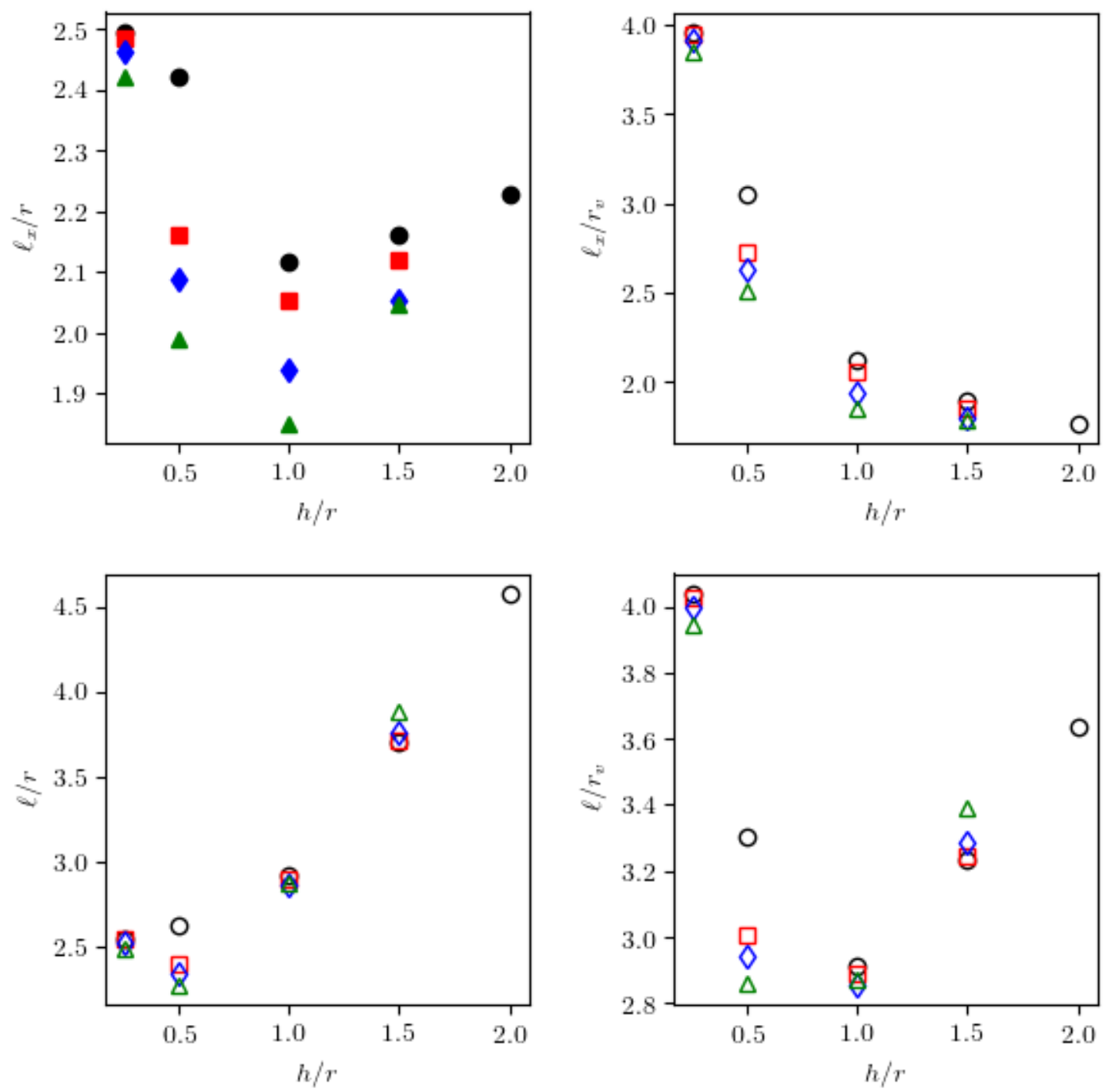

Figure 13. Critical distance $\ell_{x}$ normalised by the mean horizontal radius $r$ (left) or the mean overall radius $r_{v}$ (right) vs the height-to-width aspect ratio $h / r$ for the full QG equilibria and $\delta z / h=0$ (०) $2 / 61 \square, 6 / 61 \diamond$ and $12 / 61 \triangle$ (colour online).

(2002). Figure 12 shows the spatial structure of the most amplified mode for three examples. It is plotted in the plane $\left(\theta_{t}, z\right)$. Here, $\theta_{t}$ is the travel coordinate which is defined along each contour and is an angle $0 \leq \theta_{t}<2 \pi$ proportional to the time taken by a fluid particle to travel along the contour. $\theta_{t}=0$ corresponds to the outermost edges of the vortices while $\theta_{t}=\pi$ corresponds to their innermost edges. Results show that the spatial structure of the modes is non-trivial which is not surprising considering that the vortices are strongly deformed. The modes corresponding to the instability in the narrow range for $h / r=0.5$ (see label $A$ in figure 11) and $h / r=1$ are different but share two common characteristics. First, the deformation is symmetric with respect to the horizontal plane $z=0$ separating the lower and upper vortices located at $-0.5 \leq z<0$ and $0<z \leq 0.5$ respectively. Second, for a given $z$, there is only two changes of sign of the deformation along the contour, $0 \leq \theta_{t} \leq 2 \pi$, indicating a contribution of the first azimuthal mode $m=1$. The vertical structure is however different with a maximum deformation in the mid-horizontal cross-section for the oblate V-state with $h / r=0.5$ and near the region where the vortices are at their closest for $h / r=1$. In both cases, the deformation 

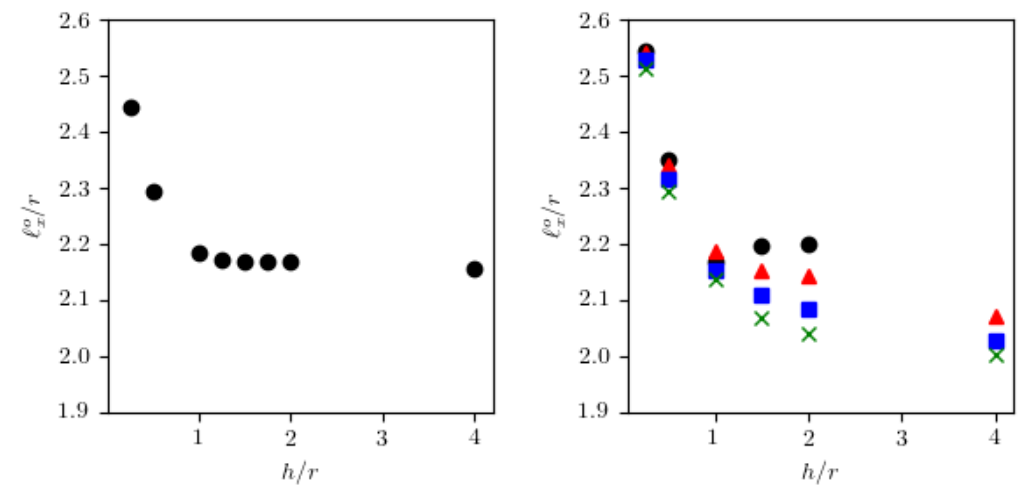

Figure 14. Normalised horizontal overlapping critical distance $\ell_{x}^{o} / r$ for equilibrium vortices: ellipsoidal equilibrium for $\delta z=0$ (left) and full QG equilibria (right) for $\delta z=0$ (black), $\delta z / h=2 / 61$ (red), 6/61 (blue), 12/61 (green). There is horizontal overlapping between the two vortices if $\ell_{x} / r<\ell_{x}^{0} / r$, no overlapping otherwise (colour online).

is very localised. On the other hand, the spatial structure of the most amplified mode for the oblate vortices in the second region of instability (for smaller $\ell_{x}$, see label $B$ in figure 11) is different. The deformation is anti-symmetric with respect to $z=0$ and the mode projects on $m=2$ in the azimuthal direction as the deformation changes four times of sign.

The critical distance $\ell_{x}$ where the peak of instability is observed is shown in figure 13 for various aspect ratios $h / r$ and vertical offsets $\delta z$. We normalise $\ell_{x}$ by either the mean horizontal radius $r$ or by the mean radius $r_{v} \equiv(3 V / 4 \pi)^{1 / 3}=r(h / r)^{1 / 3}$, where $V$ is the vortex volume. Although $r$ is, a priori a more natural scaling for normalising a horizontal separation between the vortices, $r_{v}$ is meaningful as it is a measure the vortices overall volume (and strength). When normalised by $r_{v}$ the critical distance $\ell_{x} / r_{v}$ decreased monotonically both when the vortex height-to-width aspect ratio $h / r$ is increased and when the vertical offset $\delta z$ between the vortices is increased. The latter is simply due the decrease in the intensity of the interaction as vortices are separated in the vertical direction. The former is not as simple as the critical distance normalised by the mean horizontal radius $\ell_{x} / r$ is not a monotonic function of $h / r$. The figure also shows the full critical distance $\ell=\sqrt{\ell_{x}^{2}+\ell_{z}^{2}}$ normalised by $r$ and $r_{v}$ as functions of $h / r$. Both $\ell_{x} / r$ and $\ell / r_{v}$ exhibit a minimum for $h / r \simeq 1$ indicating that these vortices are robust, as an unstable mode can only be found for smaller a relative separation distance compared to the configurations. Note that a complete study of ellipsoidal vortices under linear external strain has been conducted in Reinaud et al. (2003), where it is shown that ellipsoids with $h / r \simeq 0.8$ can exist in equilibrium for the largest value of external strain on average. The robustness of vortices with height-to-width aspect ratio of $h / r \simeq 1$ explains their prevalence in QG turbulence.

Figure 14 shows the normalised horizontal separation $\ell_{x}^{o} / r$ below which the vortices of the V-state overlap. By symmetry, the two vortices overlap the innermost edge of the vortex on the right is located at some $x<0$. For an ellipsoidal vortex $x$-coordinate of the innermost edge corresponds to $X-\sqrt{\mathcal{B}_{11}}$, while this corresponds simply the minimum $x$-coordinate of the nodes discretising the boundary of the vortex on the right for the full QG V-states. Recall that upright-standing spheroids overlap if $\ell_{x} / r \leq 2$. Results show that the overlapping distance decreases with $h / r$. This is due to the fact that the relative amplitude of the horizontal deformation of the $\mathrm{V}$-states decreases as $h / r$ increases. We note that vortices only overlap when they are close together. This also corresponds to the regions where $\theta$ is small: the vortices are nearly upright-standing. This is particular true for the full QG V-states. Oblate vortices can be elongated in the $x$-direction, see figure 9 . For the full QG V-states, results indicate that $\ell_{x}^{o} / r \rightarrow 2$ as $h / r$ increases. For the ellipsoidal V-states, $\ell_{x} / r$ decreases more slowly as $h / r$ increases and $\ell_{x}^{o} / r \simeq 2.16$ for $h / r=4$. The difference is due to the fact that the 

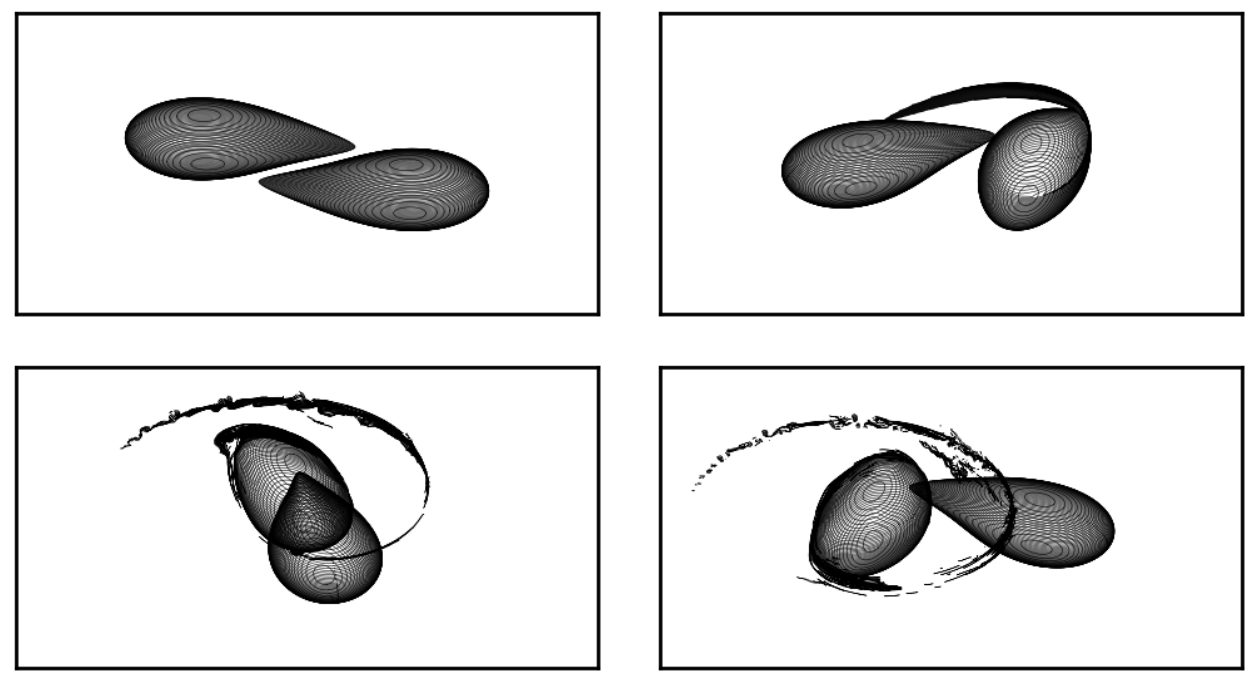

Figure 15. Evolution (full QG dynamics) of an unstable full QG equilibrium for $h / r=0.25$ and $\ell_{x} / r=2.5$. Orthographic view on the vortex bounding contours at an angle of $65^{\circ}$ from the vertical direction at $t=0,2.5,10$ and 15 .
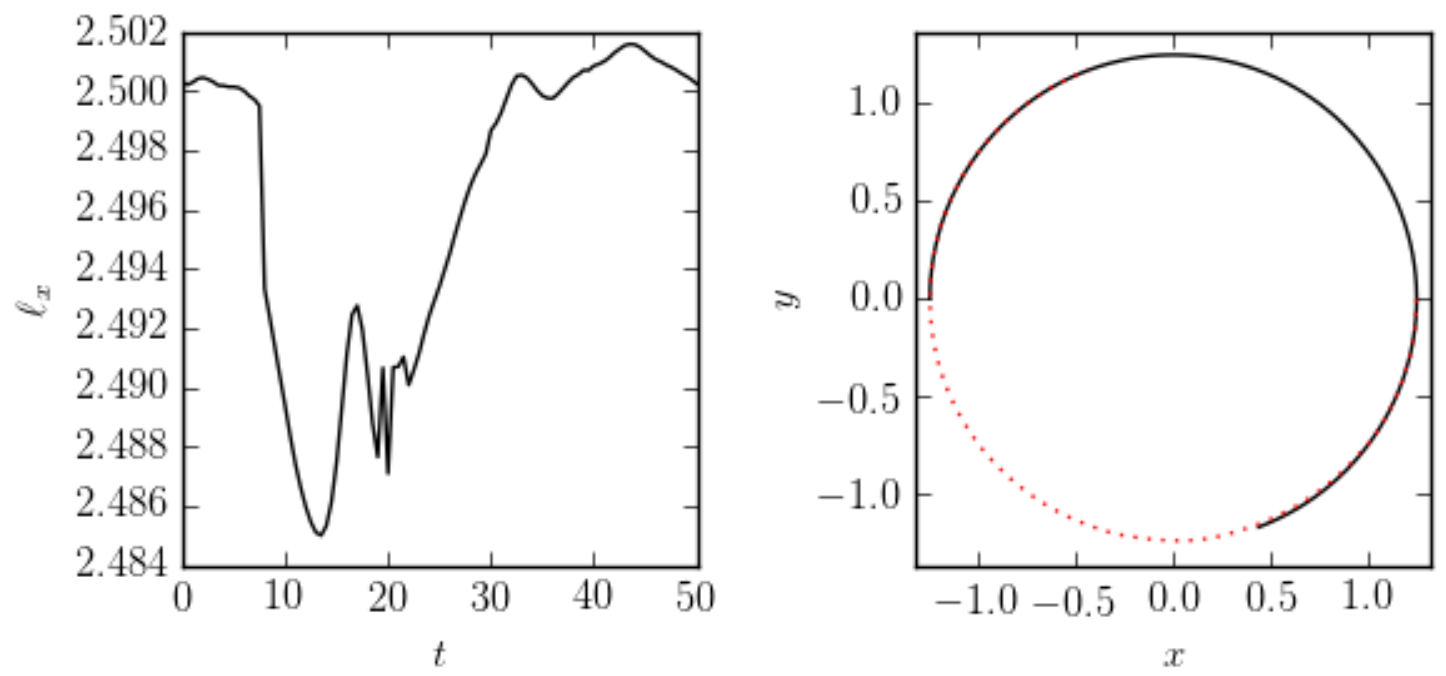

Figure 16. Evolution (full QG dynamics) of the distance between the centres of the two main vortices for an unstable full QG equilibrium with $h / r=0.25$ and $\ell_{x} / r=2.5$ (left). Trajectories of the centre of the two main vortices (right).

ellipsoidal V-states are more tilted.

We next illustrate the nonlinear evolution of unstable full QG V-states. In the first example, we present the evolution of the last $\mathrm{V}$-state obtained for $h / r=0.25$ and $\delta z=0$ already shown in figure 9 . Results are shown in figure 15 in a reference frame steadily rotating at the angular velocity $\Omega$ of the $\mathrm{V}$-state. This means that in absence of instability, the vortices would be steady in this reference frame. The instability results in the vortices being put out of their initial alignment. A filament of PV sheds from one of the vortices as asymmetry between the two vortices grows. The loss of material by one of the vortices increases the asymmetry between the two vortices.

The distance between the two main vortices remains almost constant, varying only by approximately $6.5 \%$ as seen on the left panel of figure 16 . The deformation of the vortices combined with growing asymmetry between the two slightly modify the angular velocity of 

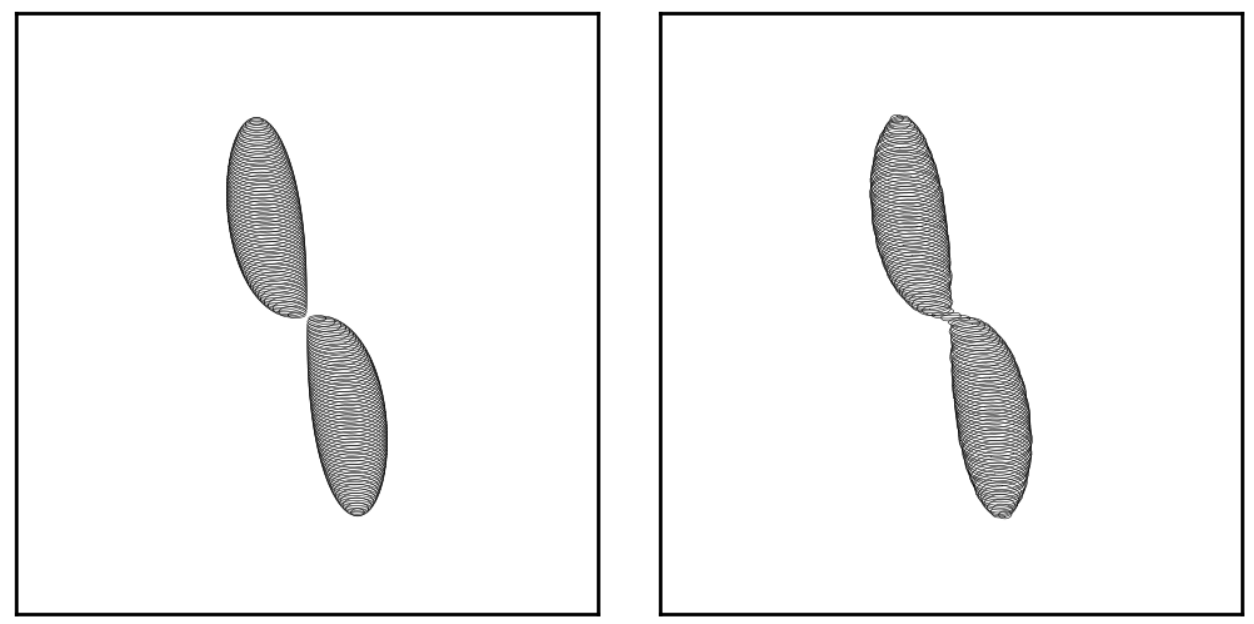

Figure 17. Evolution (full QG dynamics) of an unstable full QG equilibrium for $h / r=2$ and $\ell_{x} / r=2.23$. Orthographic view on the vortex bounding contours at an angle of $65^{\circ}$ from the vertical direction at $t=0$ and 50 .

the vortex pair. Yet, as seen from the right panel of figure 16, the trajectory of the vortex centres, shown in the reference frame rotating at the equilibrium angular velocity, remain circular.

For the second example, we investigate the evolution of an unstable pair of vortices for $h / r=2$ and $\ell_{x} / r=2.23$. Recall that the vortex pair is only unstable in a very narrow range of $\ell_{x}$. Results are shown in figure 17 . Here, the instability has rather little effect on the equilibrium as the vortices almost do not move. The effect of the instability is first very local: the bottom contours of the top vortex align with the top contours of the bottom vortex slightly deform and align. This local deformation is accompanied by waves which travel horizontally along the contours and also travel up and down each vortex. Nonetheless the nonlinear interaction remains very weak. This is generic of the interactions between prolate vortices.

Finally, we consider the nonlinear evolution of $\mathrm{V}$-state for $h / r=0.5$ and $\ell_{x} / r=2$ (see label $B$ in figure 12). This state corresponds to an unstable equilibrium in the second unstable region for oblate vortices. Results are presented in figure 18. From figure 12 (middle panel), the most amplified mode corresponds to a mode of deformation which is maximum where the vortices are close together $(z \simeq \pm 0.068)$ and their innermost edges $\left(\theta_{t} \sim \pi\right)$. In the nonlinear regime, the deformation becomes asymmetric and the bottom vortex elongates while the top vortex becomes more compact as a consequence of conservation of $J$. Eventually the bottom vortex splits into two vortices asymmetrically. The largest part of the bottom vortex nearly aligns with the top vortex.

\section{Interaction between upright-standing QG spheroidal vortices}

We finally complement the study by considering the nonlinear evolution of two identical, nonequilibrium, upright-standing spheroids of uniform potential vorticity using contour surgery. We restrict attention to the case $\delta z=0$. The fluid domain containing PV is mapped by 100 horizontal layers, such that each vortex is spanned vertically by 50 horizontal layers. The initial conditions consist in two identical, upright-standing spheroidal vortices of a prescribed aspect ratio $h / r$ and whose centres are separated by a prescribed horizontal distance $\ell_{x}$. We consider $0.25 \leq \ell_{x} / r \leq 4$, with an increment of $\Delta \ell_{x} / r=0.25$ between cases. It should be noted that the case $\ell_{x}=0$ offers no interest as the vortices are then in stable equilibrium (Dritschel, 1988b). The vortex aspect ratios $h / r$ considered are $h / r=0.25,0.5,1,1.5,2,3$ 

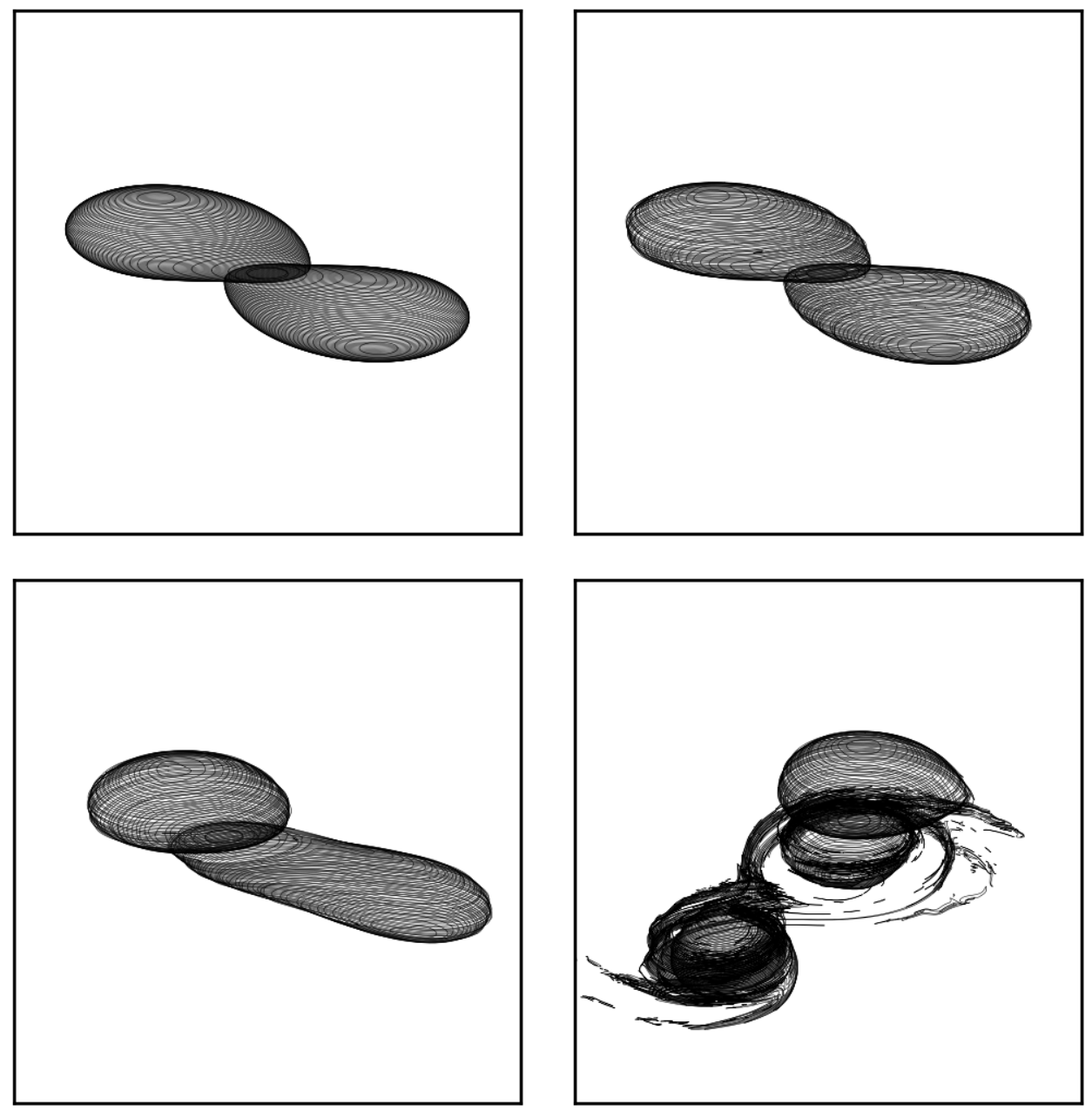

Figure 18. Evolution (full QG dynamics) of an unstable full QG equilibrium for $h / r=0.5$ and $\ell_{x} / r=2$. Orthographic view on the vortex bounding contours at an angle of $60^{\circ}$ from the vertical direction at $t=0,75,100$ and 125 .

and 4. The vortex pair occupy the region $0 \leq z \leq 1$ in the vertical direction. Since $\delta z=0$, this means $h=0.25$ for both vortices. Each vortex has a PV set to $q= \pm 2 \pi$. Recall that an isolated uniform PV sphere has a turnover period of $T_{t}=6 \pi / q=3$ here. All simulations are run until $T_{\max }=200 \simeq 67 T_{t}$.

We first analyse two criteria which categorise the overall behaviour of the vortex pairs. First, we identify all contiguous volumes of PV (i.e. all vortices). The analytical initial conditions consist of two spheroidal vortices. In theory, at $t=0$ they can only form a single contiguous volume in the limiting case $\ell_{x}=0$ (and $\delta z=0$, assumed throughout the section), where they touch. Due the layerwise discrete nature of the vortex representation, the top and bottom of the spheroids are represented by finite area contours. These can overlap for $\ell_{x}$ small. Hence, the 'two' vortices can be seen as a single structure for small $\ell_{x}$. This overlapping of the two neighbouring contours of the two vortices depends on the vertical discretisation by essence. Yet, the analysis of evolution of the number of identifiable structures still reveals information about the dynamics of the flow. To determine if two contours overlap, we determine whether any node discretising one contour is located inside the area bounded by the second contour. This can be done by calculating the winding number, by contour integration, of the second contour relative to the nodes of the first contour. Figure 19 (top, left) shows the number of vortices as identified by the algorithm at $t=0$. The vortex pair is seen as forming a 

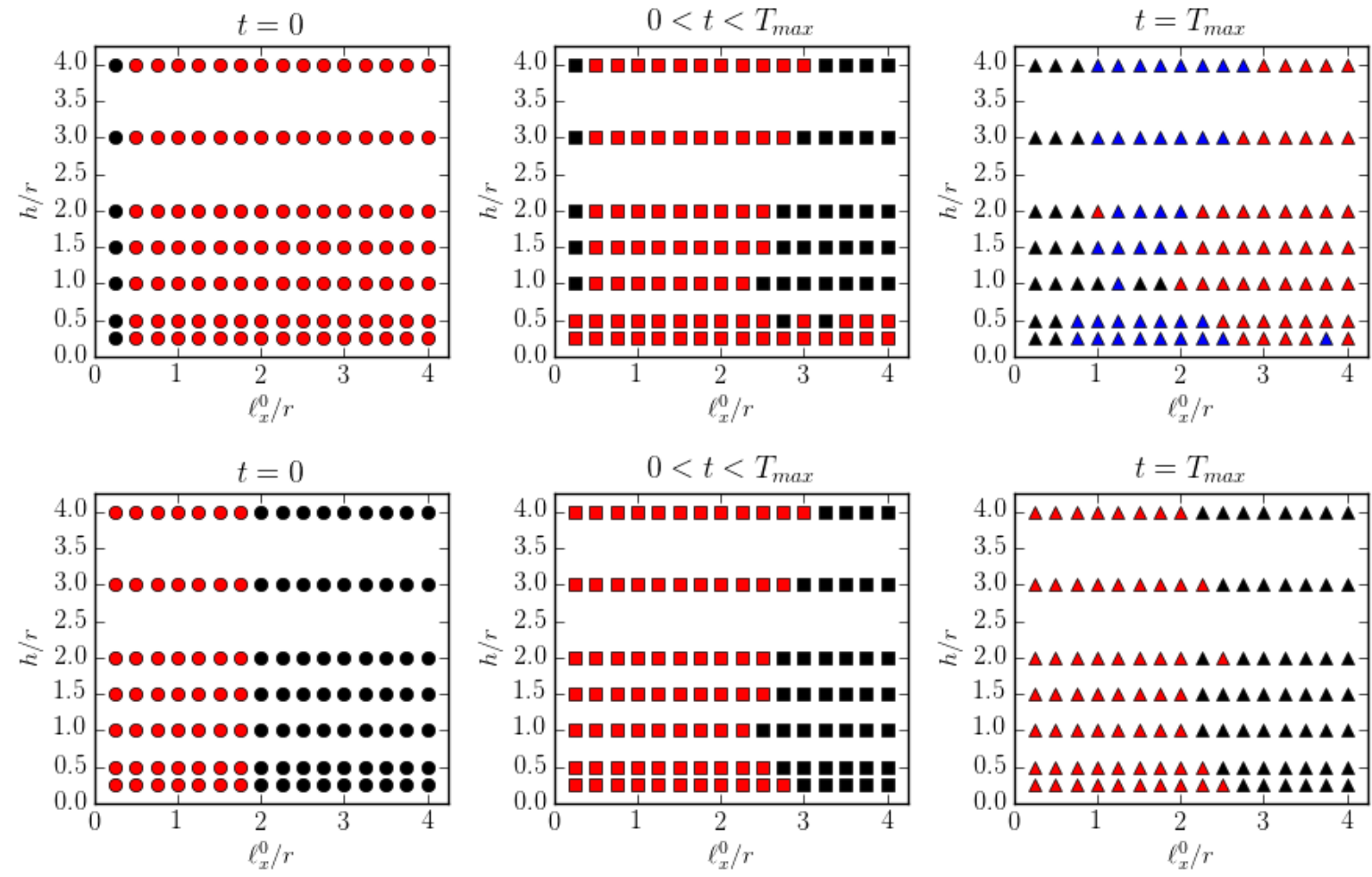

Figure 19. Non-equilibrium vortex spheroids at $t=0$ (left, circles), at intermediate time (centre, squares) and at $t=T_{\max }$ end of simulation (right, triangles). Top row: coherence of the vortex structures. Left: the vortex pair forms a single contiguous vortex structure (black symbol), the two vortices are distinct vortices (red symbol). Center: the number of identified structures remains unchanged throughout the simulation (black), the number changes (red). Blue symbol in the right column means that there are more than 2 vortices identified (satellites, debris, filaments). Bottom row: vertical alignment/horizontal overlapping of the two vortices (red symbol), no horizontal overlapping (black symbol) (colour online).

single structure for $\ell_{x} / r=0.25$ and two distinct structures otherwise. Figure 19 (top, middle) indicates whether the number of identifiable structures (including PV filaments and PV debris) has changed throughout the simulation, $0 \leq t \leq T_{\max }=67 T_{t}$. The results show that for prolate vortex pairs $h / r \geq 1$, both nearly aligned vortices $\left(\ell_{x} / r=0.25\right)$, and those well separated in the horizontal direction $\left(\ell_{x} / r \geq 3.25\right)$ do not generate any debris of filament nor separate (if they were initially contiguous for $\ell_{x} / r=0.25$ ) or align (if $\ell_{x} / r$ large). In these cases, the interaction is very weak and the vortices are robust. Figure 19 (top, right) provides information on the number of identified vortices at the end of the simulations, $t=T_{\max }$. For most intermediate horizontal separation $\ell_{x} / r$, satellite vortices, filamentary PV and PV debris are still present in the flow, indicating a stronger, more destructive interaction.

To quantify the vertical alignment associated with a horizontal overlapping of the vortices, we re-analyse the data. We first separate explicitly the fluid domain in two regions in the vertical direction: region $C$ corresponding to the bottom half region, $0<z<0.5$ and region $D$ corresponding to the top half $0.5<z<1$. Initially, exactly one vortex lie in each region. We next identify the largest vortex in each region at all time $t$. Finally we test if any contour bounding the largest vortex identified in region $C$ overlap with any contour bounding the largest vortex identified in region $D$. Results are presented in the bottom row of figure 19 . The left panel shows the initial overlapping. This consistently indicates that vortices overlap horizontally provided $\ell_{x} / r<2$, validating the detection tool. The right panel of figure 19 shows the overlapping at the end of the simulation $t=T_{\max }$. It is important to notice that this figure only provides information on the topology of the vortex configuration at a fixed time. The middle panel of figure 19 indicates if overlapping has been detected at anytime 

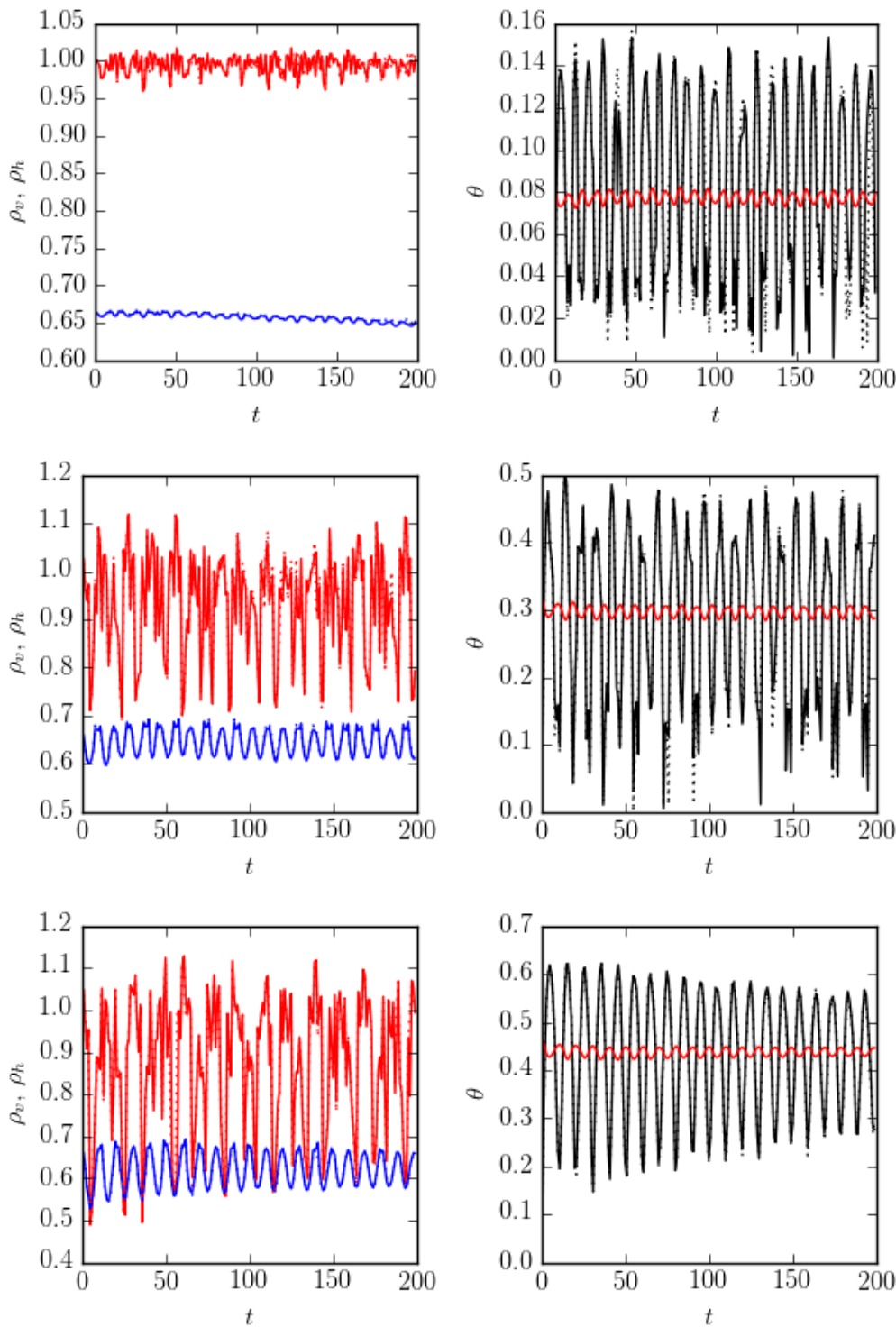

Figure 20. Left column: time evolution of the vortex horizontal and vertical aspect ratios $\rho_{h}$ (red) and $\rho_{v}=r / h$ (blue) for non-equilibrium vortex spheroids (upright-standing at $t=0$ ) for $h / r=1.5$ and $\ell_{x} / r=0.25$ (first row), $\ell_{x} / r=1$ (second row), $\ell_{x} / r=1.5$ (third row). Right column: vortex tilt $\theta$ angle (black), inter-centre angle $\phi$ (red) from the vertical direction (colour online).

during the simulations $0 \leq t \leq T_{\max }$. Results show that overall, some oblate vortices can overlap for $\ell_{x} / r>2$ for long times. Prolate vortices which do not overlap initially do not overlap for long period of time, or if they do it is only periodically.

We next illustrate some typical vortex behaviour during the interaction on a few selected examples. Figure 20 shows the evolution of the horizontal and vertical vortex aspect ratios, $\rho_{h}$ and $\rho_{v}$ respectively, as well as the tilt angle $\theta$ and inter-centre angle $\phi$ for $h / r=1.5$ and $\ell_{x} / r=0.25,1$, and 1.5. We first observe oscillations for both the angle and the aspect ratios at the same main frequency. The oscillations of the horizontal aspect ratio appear however to be noisier. The non-equilibrium vortices pulsate and this quasi-periodic deformation are associated with the oscillation of the tilt angle $\theta$. In all cases, $\theta(t=0)=0$ as the vortices are initially upright-standing spheroids. The angle $\theta$ however oscillates for $t>0$ around the inter-centre angle $\phi$, indicating that the vortices attempt to align slantwise. It should be noted however, $\theta$ cannot simply converge to $\phi$ as the system is non diffusive, hence there is no viscous 

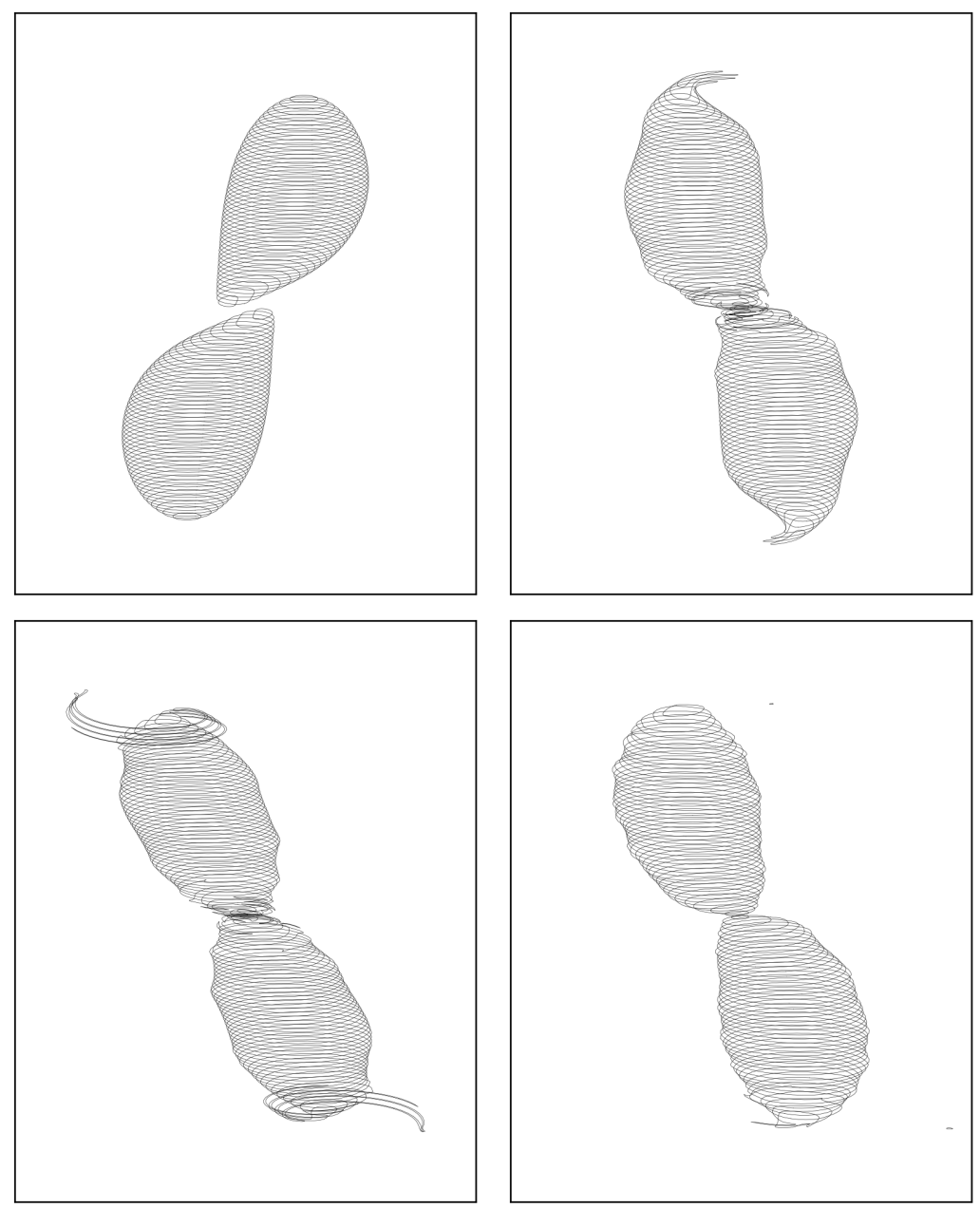

Figure 21. Evolution (full QG dynamics) of a pair of initially upright-standing vortices with $h / r=1.5$ and $\ell_{x} / r=1.5$. Orthographic view on the vortex bounding contours at an angle of $75^{\circ}$ from the vertical direction at $t=2,13,17$, and 200 .

damping of the oscillations. Oscillations can potentially be damped by the loss of energy of the main vortices to filaments. For prolate vortices, filamentation occurs on the vertical edges of the vortex pair, as (Rossby) waves along the contours (PV jumps) steepen. The process of filamentation has been described in Dritschel (1988c). Snapshot of the vortices for $h / r=1.5$ and $\ell_{x} / r=1.5$ are shown in figure 21 and illustrate the filamentation.

We next consider the case $h / r=1$ and $\ell_{x} / r=1$. As for the previous case, the two vortices are seen, in figure 22, to initially strongly interact locally in the region where they are the closest to each other. This induces a local partial alignment of contours bounding the near edges of the vortices. Rossby waves propagate on the vortex boundary and the deformation of the vortex bounding contours become steep on the top and bottom edges of the vortex pair, generating PV filaments. This shedding of filaments away from the main structures and the deformation of the vortices allow the main structures to get closer while conserving the angular impulse $J$. The same phenomenon occurs during vortex merger. Figure 23 shows the time evolution of the horizontal distance between the vortex centres $\ell_{x}$ as a function of time as well as the trajectory of the vortex centres projected onto a horizontal plane (recall that the vortex centres do not lie in the same horizontal plane). The distance $\ell_{x}$ decreases rapidly at the early stage of the flow evolution, then oscillates around a value less than the initial value. The overall decrease of $\ell_{x}$ indicates the tendency of the vortices to align. The trajectory 

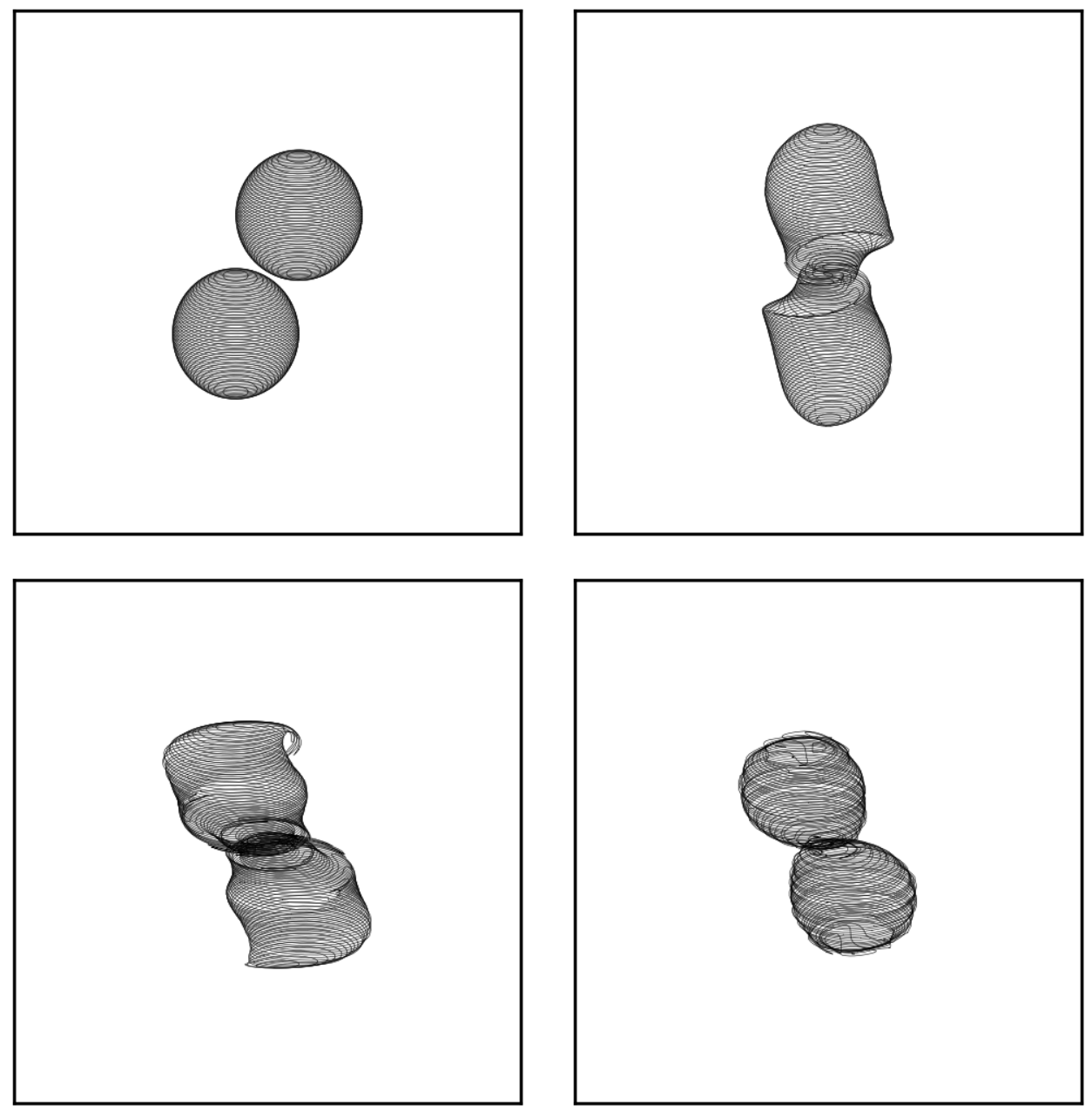

Figure 22. Evolution (full QG dynamics) of a pair of initially upright-standing vortices with $h / r=1$ and $\ell_{x} / r=1$. Orthographic view on the vortex bounding contours at an angle of $75^{\circ}$ from the vertical direction at $t=0,5,10$, and 50 .

of the centres remain nearly circular, with a small decrease of the mean radius. The ability of the vortices to get closer horizontally is associated with their ability to deform and to shed PV away from the main structures. This is therefore associated with the strength of the interaction between the vortices and the strain they induce onto each other. This varies with the distance $\ell_{x}^{0}=\ell_{x}(t=0)$ separating the vortices initially. The evolution of $\ell_{x}$ for $h / r=1$ and $\ell_{x}^{0} / r=0.25 n, 1 \leq n \leq 16$ is shown in figure 24. Results show $\ell_{x}$ oscillates for $\ell_{x}^{0} / r \leq 3$. Oscillations almost disappear for $\ell_{x}^{0} \geq 3$. In general, $\ell_{x}$ decreases from its initial value $\ell_{x}^{0}$ except for the case $\ell_{x}^{0} / r=3.75$ where a very small increase of the separation distance is observed. In this case the interaction is non-destructive (no PV filaments not PV debris are created), but the vortices deform and tilt (results not shown). The maximum decrease of the mean value of $\ell_{x}$ is observed for $1 \leq \ell_{x}^{0} / r \leq 2$, which corresponds to the range where the equivalent $\mathrm{V}$-states are the most deformed, see figures 4 and 8 . This indicates a strong strain induced by the vortices on each other. The evolution of $\ell_{x}$ as a function of time exhibits a generic behaviour, as shown in figure 24: an initial abrupt changed corresponding to the initial adaption of the non-equilibrium, up-right standing spheroidal vortices to a nearby equilibrium. Then the vortices deform quasi-periodically around this pseudo-equilibrium.

The trends on the evolution of $\ell_{x}$ can therefore be summarised by measuring the departure of an averaged $\ell_{x}$ after the initial adaptation stage from its initial value. For that purpose we 

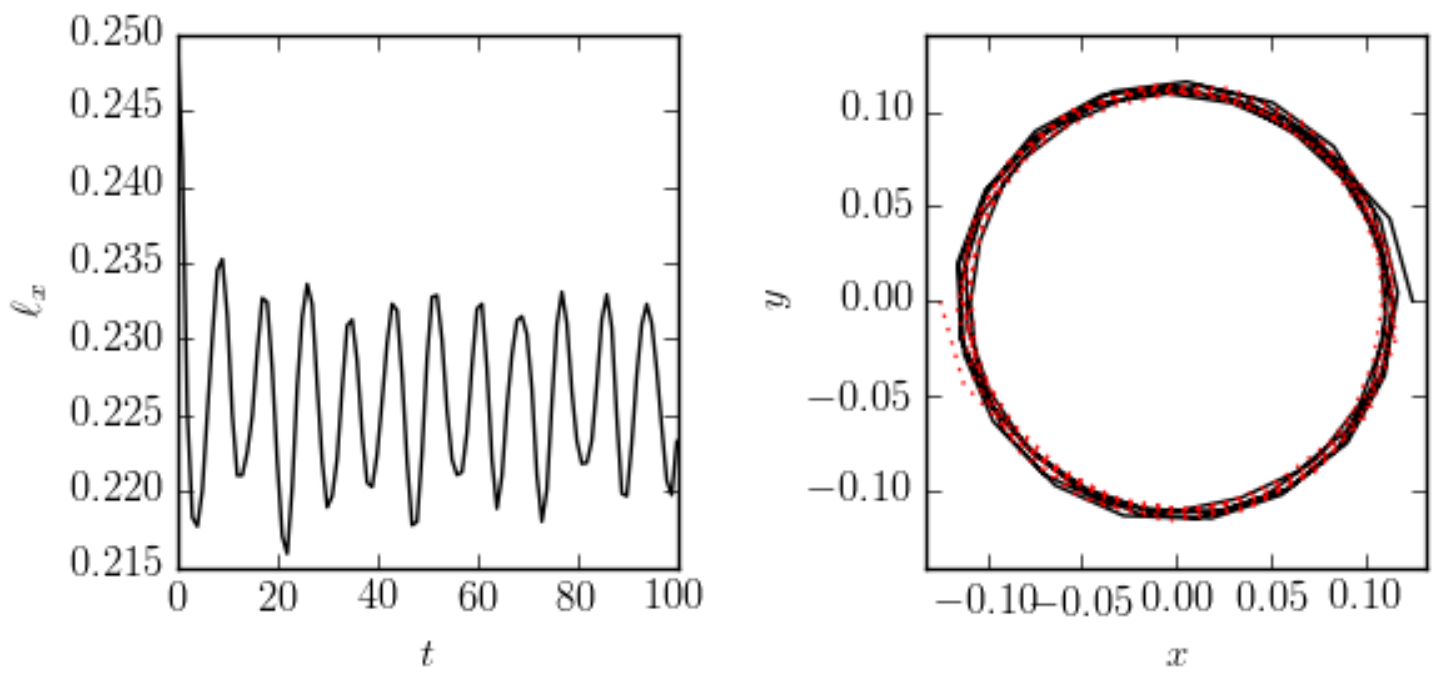

Figure 23. Evolution (full QG dynamics) of the horizontal distance between the centres of the two main vortices for of a pair of initially upright-standing vortices with $h / r=1$ and $\ell_{x}^{0} / r=1$ at $t=0$ (left). Trajectories of the centre of the two main vortices (right).

define

$$
\overline{\ell_{x}}=\frac{1}{\Delta T} \int_{T_{\max }-\Delta T}^{T_{\max }} \ell_{x}(t) \mathrm{d} t
$$

which $\Delta T=150=2 T_{\max } / 3$. This value provides, in practice, the largest averaging time window in the quasi-periodic oscillations of $\ell_{x}$ over the full parameter space. Figure 25 provides the value of $\overline{\ell_{x}}-\ell_{x}^{0}$ for all values of $h / r$ and $\ell_{x}^{0}$ investigated. For $h / r \geq 1$, (except $h / r=1$, $\left.\ell_{x}^{0} / r>2\right), \overline{\ell_{x}}<\ell_{x}^{0}$ indicating a tendency to alignment. This tendency is maximum for $1 \leq$ $\ell_{x}^{0} / r \leq 2$. Yet, in all these cases $\left(\overline{\ell_{x}}-\ell_{x}^{0}\right) / r$ remains small. This is in contrast with the cases $h / r=0.25, \ell_{x}^{0} / r \leq 2$ for which $\left(\overline{\ell_{x}}-\ell_{x}^{0}\right) / r$ is close to $-\ell_{x}^{0} / r$ indicating that $\overline{\ell_{x}}$ is very small. In that case, the two main vortices are nearly aligned. This is also true, albeit to a lesser extent, for $h / r=0.5, \ell_{x}^{0} / r \leq 2$. As already mentioned, conservation of the angular impulse means that alignment can only occur if a significant amount of PV is ejected away from the main structure so compensate for the migration of PV towards the centre of the domain where the vortices align. This can be achieved if the vortices strongly interact. This is the case if the initial non-equilibrium attempt to adapt to a nearby unstable equilibrium. The study of the $\mathrm{V}$-states indicates that this is likely to be the case for moderately horizontally offset oblate vortices. It should also be noted that for fixed volume vortices, and given $\ell_{x} / r$, the minimum distance between any point of the first vortex and a point to second vortex can be minimised by decreasing $h / r$, hence oblate vortices are expected to interact more strongly.

We next illustrate the interaction between two oblate vortices where the vortices nearly align. The vortices have height-to-width aspect ratio $h / r=0.25$ and are horizontally separated by $\ell_{x}^{0} / r=1.75$ at $t=0$. This case corresponds to the case for $h / r=0.25$ for which the largest number of filaments and PV debris are formed during the nonlinear flow evolution. Snapshots of the vortices are presented in figure 26. Two large tongues of PV form and stretch away form the domain centre. This tongues of PV rotate more slowly than the structures at the centre to which they are attached and are therefore stretched. The tongues of PV are shear zone. Their stretching by the differential rotation tends to stabilise them. The stabilising effect of a strain on the PV filament has been analysed in two dimensions by Dritschel (1989b). If this stretching is not intense enough, the elongated PV tongues or filaments can roll-up and self-organise into secondary Kelvin-Helmholtz like billows, as shown in figure 26. At the end of 

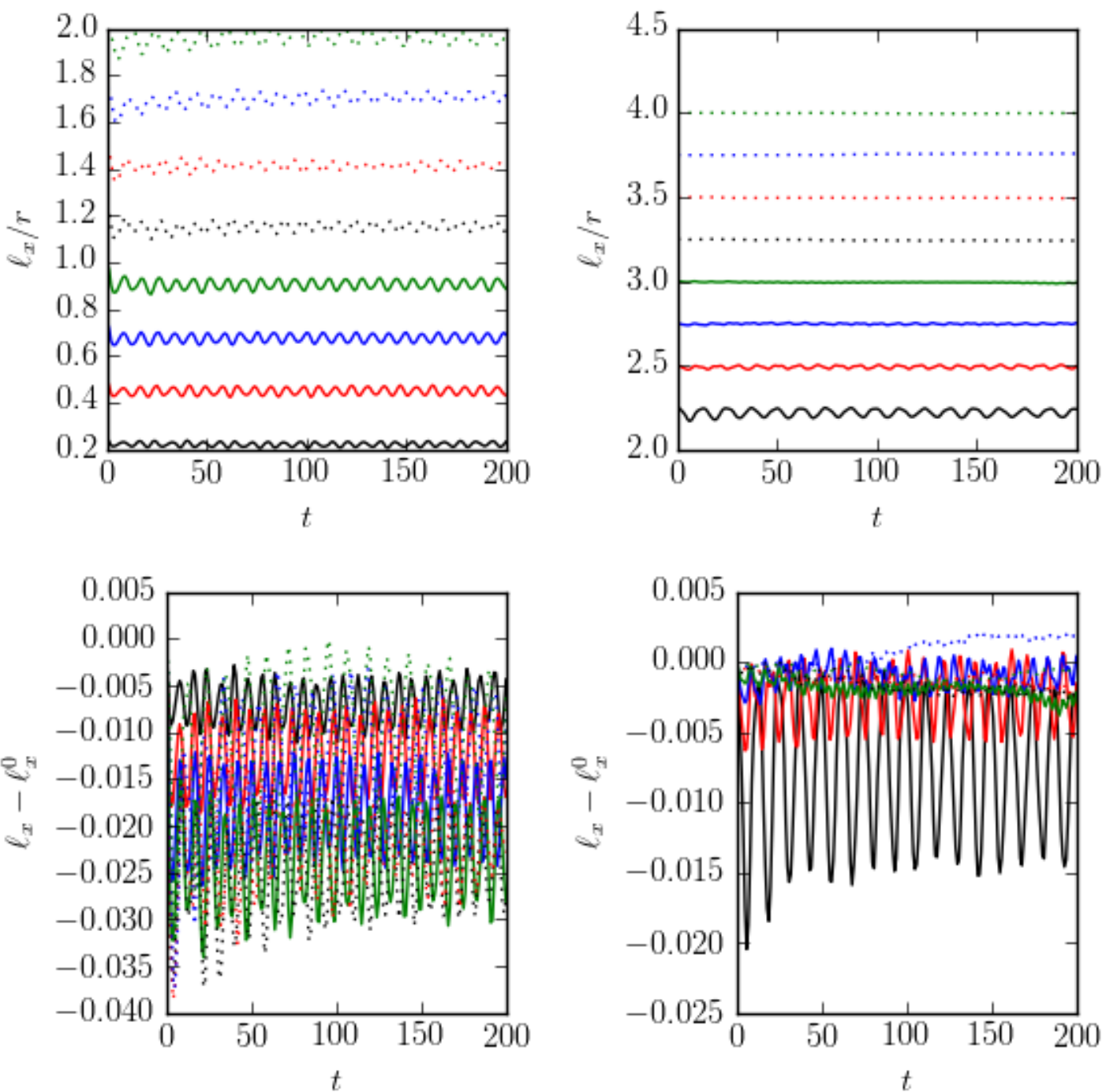

Figure 24. Evolution (full QG dynamics) of the horizontal distance $\ell_{x}$ between the centres of the two main vortices for of a pair of initially upright-standing vortices with $h / r=1$ and $\ell_{x}(t=0) / r \equiv \ell_{x}^{0} / r=0.25 n, 1 \leq n \leq 8$ (left) and $9 \leq n \leq 16$ (right).

the simulation the PV distribution consist in two main large structures nearly aligned next to the domain centre surrounded by a sea of secondary small scales vortices and PV debris. The alignment of the main structures can be clearly seen from the steep decrease of the horizontal distance separating the two main vortices $\ell_{x}$ as shown in figure 27 . The trajectory of the vortex centres is also presented in figure 27 . The small loops that can be observed in the early trajectory of the vortex centres are due to the formation of the PV tongues which moves the vortex centres. In this case, the vortices shed a significant amount of filamentary PV. This induces a cascade in the length scales present in the flow. Due to the Lagrangian, unbounded and grid-free nature of the simulations, computing energy spectra, though possible, is not the natural way to describe the distribution of the flow properties in physical space. It is more natural to describe the properties of the vortices or more generally contiguous regions of PV present in the flow. To characterise the distribution of the some of the flow properties across the physical scales, we first associate a length scale $r_{v}$ to all identifiable coherent structures or contiguous regions of PV. This length scale is simply $r_{v}=(3 V /(r \pi))^{1 / 3}$, where $V$ is the volume of each contiguous region. We then bin the vortex lengths scales into 20 bins of radius range 

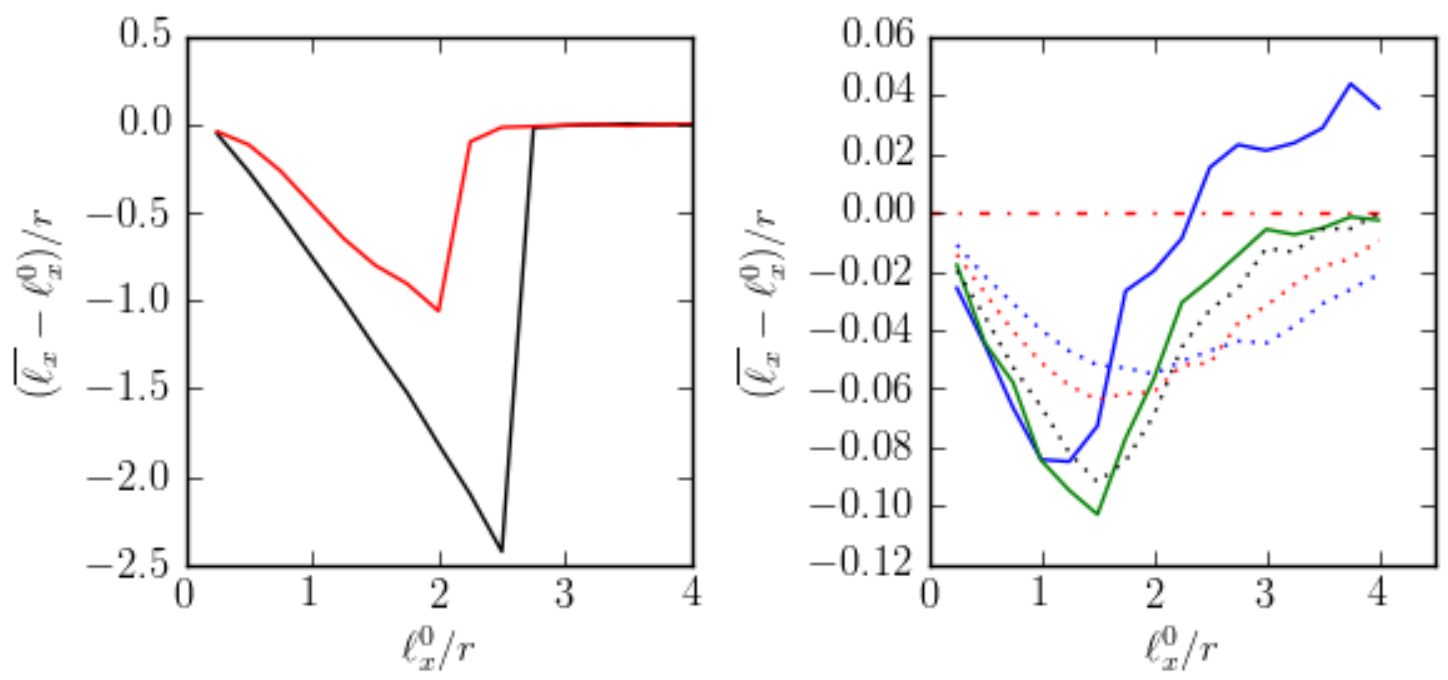

Figure 25. Departure of the normalised mean value of $\ell_{x}$ over $50 \leq t \leq 200, \overline{\ell_{x}}$ from its normalised initial value $\ell_{x}(t=0) \equiv \ell_{x}^{0}$ vs $\ell_{x}^{0} / r$ for $h / r=0.25$ (left, black solid line), 0.5 (left, red solid line), 1 (right, blue solid line), 1.5 (right, green solid line), 2 (right, black dotted line) 3 (right, red dotted line) and 4 (blue dotted line) (colour online).
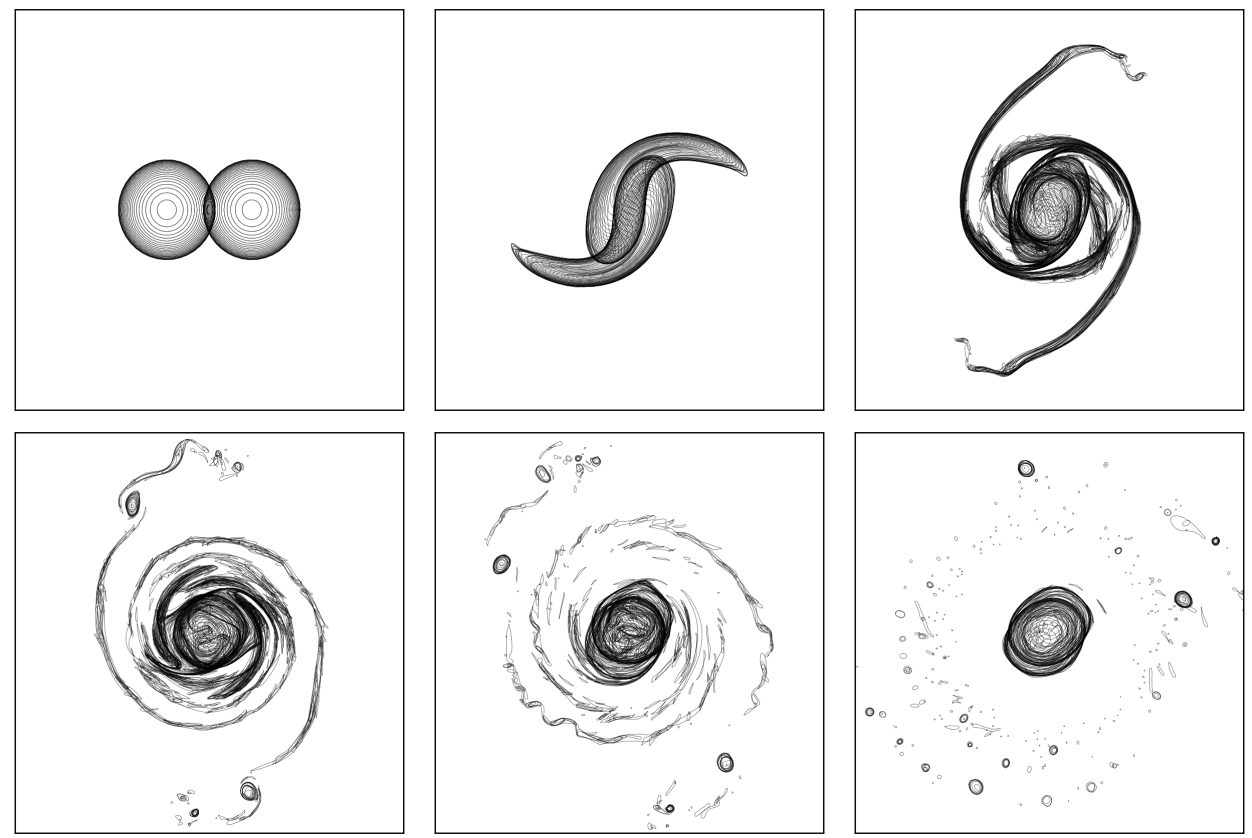

Figure 26. Evolution (full QG dynamics) of a pair of initially upright-standing vortices with $h / r=0.25$ and $\ell_{x}^{0} / r=1.75$. Top view on the vortex bounding contours at $t=0,6,22,40,60$ and 200.

between $r_{v}=0$ and $r_{v}^{\max }$. The maximum radius $r_{v}^{\max }$ is the length scale associated with the total volume of PV at $t=0$. Then, we first calculate the sum of the cube of the vortex length scales contained in each bin, which simply give a measure of the volume of PV contained in each length scale bin. Results are shown in figure 28. Initially, all the volume of PV in contained within the length scale associated with the two vortices. During the early stages of the flow evolution, the vortices deform and they lose material to PV tongues and consequently to filaments, small secondary vortices and debris. This feeds both intermediate (mostly the large filaments) and small scales of the flow. It is also accompanied by a reduction of the length scale where most of the volume is contained as the large vortices have lost material. As the 

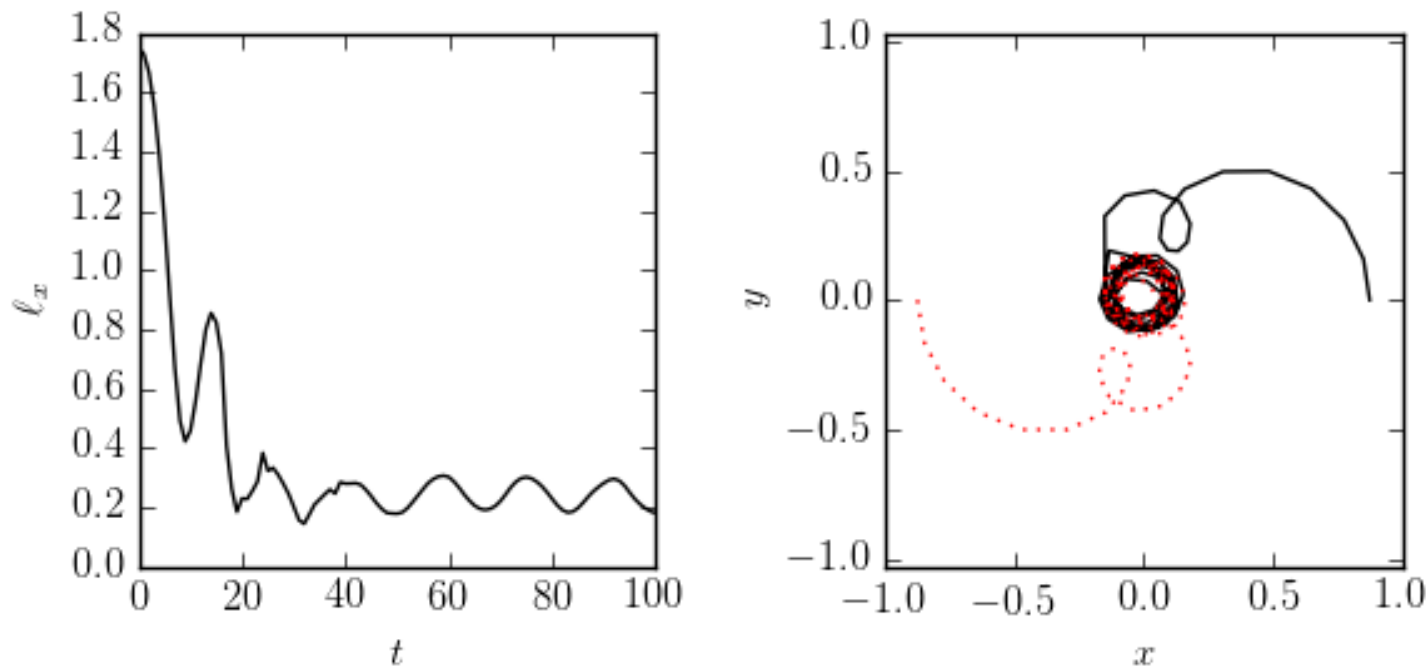

Figure 27. Evolution (full QG dynamics) of the distance between the centres of the two main vortices for of a pair of initially upright-standing vortices with $h / r=0.25$ and $\ell_{x}^{0} / r=1.75$ (left). Trajectories of the centre of the two main vortices (right).
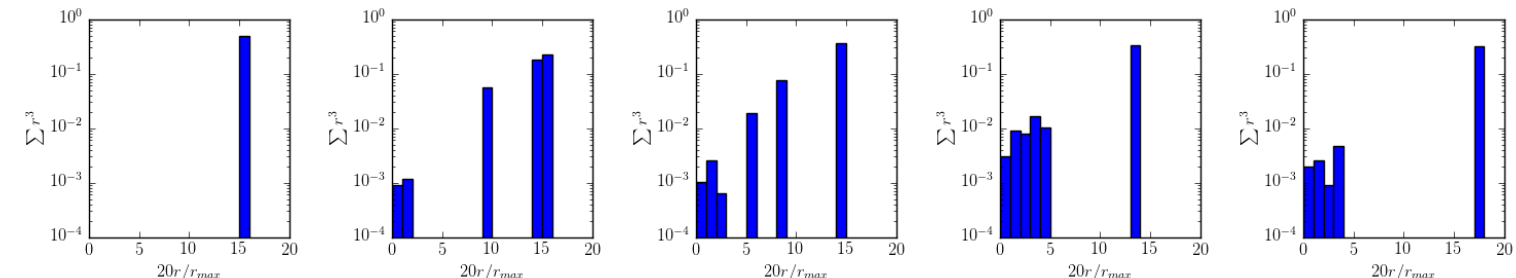

Figure 28. Evolution of a measure of the volume of PV $\sum r_{v}^{3}$ contained in given range of length scale $r_{v}$ for a pair of non-equilibrium, initially upright-standing vortices with $h / r=0.25$ and $\ell_{x}^{0} / r=2$ at $t=0,15,20,40$ and 200
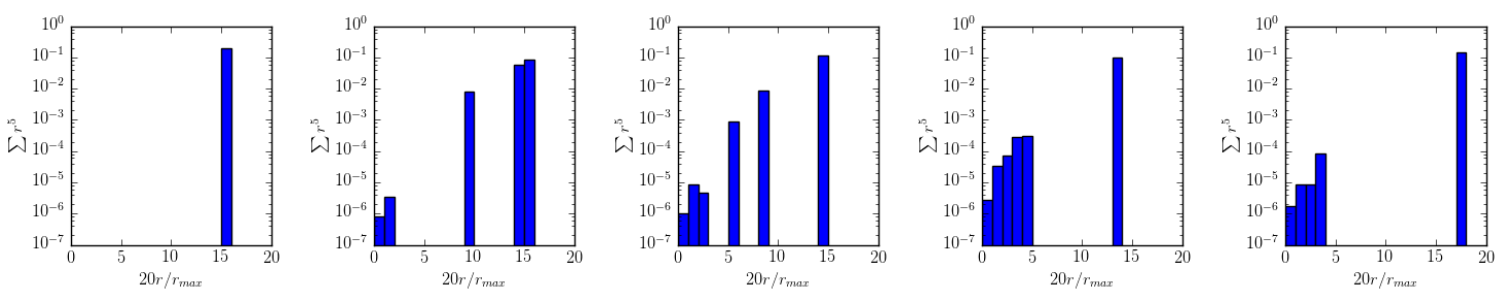

Figure 29. Evolution of an estimate of the self-energy $\sum r_{v}^{5}$ contained in given range of length scale $r_{v}$ for a pair of non-equilibrium, initially upright-standing vortices with $h / r=0.25$ and $\ell_{x}^{0} / r=1.75$ at $t=0,17,20,40$ and 200 .

flow evolves the intermediate scales disappear as the filaments break into small scale vortices. At the same time the amount of PV contained at small scale also decreases as the smallest debris are removed by Contour Surgery. On the other hand, the two main, large, structures eventually align not form a single contiguous region of PV shifting the scale at with most of the volume of $\mathrm{PV}$ is contained to a value larger than the initial value. The alignment has indeed generated a larger vortex. We also make a rough estimate of how energy is distributed across the physical length scale. To that purpose, we bin the values of $r_{v}^{5}$. The value of $r_{v}^{5}$ gives a rough estimate of the self-energy contained in a structure of mean radius $r_{v}$. Indeed, 
the self-energy of a spherical vortex of radius $a$ and uniform PV $Q_{0}$ is

$$
E=-\frac{1}{2} \iiint_{V} q \varphi \mathrm{d}^{3} \boldsymbol{x}=\frac{4 \pi}{15} Q_{0}^{2} a^{5} .
$$

It should be noted that using $r_{v}^{5}$ as a way to estimate the energy disregards the energy associated with the interaction between the vortices. Yet, this provides a very simple diagnostic tool to derive some general tendencies. It is also consistent with associating a single length scale to the structures, equivalent to the radius of a sphere of same volume. By the end of the simulation, most of the energy has been transferred to a larger scale, an inverse energy cascade, while some smaller structure carrying little energy have also been formed. These low-energy structures nonetheless carry enstrophy to smaller scales in a direct entrophy cascade.

\section{Conclusions}

We have revisited the problem of alignment of two three-dimensional, quasi-geostrophic vortices in a continuously stratified rotating fluid. We have first determined V-states using both an approximate model where vortices are modelled by ellipsoids and using the full QG dynamics.

For the case $h / r=0.25$, the branch of V-states stemming from infinitely distant uprightstanding spheroids stops before the vortices are fully aligned. There should be another disconnected branch of V-states for smaller separation distances but it could not be determined in practice. All nonlinear simulation starting non-equilibrium upright-standing spheroids for $h / r=0.25$ and small (but not zero) $\ell_{x}$ proved to be destructive. This suggests that the equivalent $\mathrm{V}$-states for small $\ell_{x}$ are unstable. It is indeed the case for $h / r=0.5$ where we have found full QG unstable V-states for small $\ell_{x}$.

For prolate vortices, all V-states are stable apart from a very narrow part of the parameter space. This narrow unstable range is found in both approaches, indicating that the instability observed is genuine and a not numerical artefact. Numerical experiments show that the instability is non-destructive in the nonlinear regime. In these cases, the instability is not clearly linked to ends of individual branches of solutions and the transition between two distinct branches. Indeed all integral quantities such as the total energy $E$, the angular impulse $J$, the angular velocity $\Omega$ as well as the second order geometrical moments measured by the best fitted ellipsoid semi-axis lengths $a, b$ and $c$ are smooth functions of $\ell_{x}$ across the unstable region.

It is nonetheless possible that different branches of $\mathrm{V}$-states exists and that for a given vortex volume, height-to-width aspect ratio and horizontal separation, multiple steady states may exist. We performed a similar (non detailed) analysis where we have replaced the spheroidal vortices by cylindrical vortices. It is clear that the branch $\mathrm{V}$-states stemming from two uprightstanding vortices located far apart $\left(\ell_{x} \rightarrow \infty\right)$, followed by moving the vortices closer together, and the branch of $\mathrm{V}$-states stemming from the two cylindrical vortex initially aligned $\left(\ell_{x}=0\right)$, followed by moving the vortices apart are two distinct branches as shown in Appendix B. Appendix B shows that the branch stemming from infinity stops for a finite $\ell_{x}$ where the vortices have formed sharp inner corner. The vortices do not overlap. There exists however a connected and distorted V-state for the same horizontal separation which belongs to the branch stemming from $\ell_{x}=0$.

For V-states stemming from spheroidal vortices, we may say that overall, and except for oblate vortices for small but not zero $\ell_{x}, \mathrm{~V}$-states are stable. The vortices of the $\mathrm{V}$-states have therefore no mechanism eject PV away from the domain centre and cannot align. Alignment is by large achieved when the vortices are initially not in mutual equilibrium. The alignment becomes a consequence of the arbitrary choice of initial conditions. Using spheroidal vortices is 
an ad hoc simple assumption but has no real physical justification for close range interactions. Alignment of oblate vortices is different as the equivalent V-states can be unstable.

Nonetheless, alignment is observed in practice. In a real situation however, pairs of vortices are not isolated and are submitted to the influence of other vortices and flow structures surrounding them. This external influences can push the vortices closer together. Vortex alignment for a vortex pair subject to a weak external strain field should be addressed.

\section{References}

Bambrey, R.R., Reinaud, J.N. and Dritschel, D.G., Strong interactions between two co-rotating quasigeostrophic vortices. J. Fluid Mech., 2007, 592, 117-133.

Chandrasekhar, S., Ellipsoidal Figures of Equilibrium, 1969 (New York: Dover).

Corréard, S. and Carton, X., Vertical alignment of geostrophic vortices: on the influence of the initial distribution of potential vorticity; in Simulation and identification of organised structures in flows, edited by J. Sorensen, E. Hopfinger and N. Aubry, Vol. 52, 1998, pp. 191-200.

Dijkstra, H.A., Dynamical oceanography, 2008 (Berlin Heidelberg: Springer-Verlag).

Dritschel, D.G., Contour surgery: a topological reconnection scheme for extended integrations using contour dynamics. J. Comput. Phys., 1988a, 77, 240-266.

Dritschel, D.G., Nonlinear stability bounds for inviscid, two-dimensional, parallel or circular flows with monotonic vorticity, and the analogous three-dimensional quasi-geostrophic flows. J. Fluid Mech., 1988b, 191, $575-581$.

Dritschel, D.G., The repeated filamentation of two-dimensional vorticity interfaces. J. Fluid Mech., 1988c, 194, $511-547$.

Dritschel, D.G., Contour dynamics and contour surgery: numerical algorithms for extended, high-resolution modelling of vortex dynamics in two-dimensional, inviscid, incompressible flows. Computer Phys. Rev., 1989a, 10, $77-146$.

Dritschel, D.G., On the stabilization of a two-dimensional vortex strip by adverse shear. J. Fluid Mech., 1989b, 206, 193-221.

Dritschel, D.G., Vortex merger in rotating stratified flows. J. Fluid Mech., 2002, 455, 83-101.

Dritschel, D.G., Reinaud, J.N. and McKiver, W.J., The quasi-geostrophic ellipsoidal vortex model. J. Fluid Mech., 2004, 505, 201-223.

Dritschel, D.G. and Saravanan, R., Three-dimensional quasi-geostrophic contour dynamics, with an application to stratospheric vortex dynamics. Quart. J. Roy. Meteorol. Soc., 1994, 120, 1267-1297.

Dritschel, D.G. and Waugh, D.W., Qualification of the inelastic interaction of unequal vortices in twodimensional vortex dynamics. Phys. Fluids A, 1992, pp. 1737-1744.

Ferrari, R. and Paparella, F., Compensation and alignment of thermohaline gradients in the ocean mixed layer. J. Phys. Oceanogr., 2003, 33, 2214-2223.

Haney, S., Fox-Kemper, B., Julien, K. and Webb, A., Symmetric and geostrophic instabilities in the wave-forced ocean mixed layer. J. Phys. Oceanogr., 2015, 45, 3033-3056.

Itoh, S. and Yasuda, I., Water mass structure of warm and cold anticyclonic eddies in the western boundary region of the subarctic North Pacific.. J. Phys. Oceanogr., 2010, 40, 2624-2642.

Jones, R.W., Willoughby, H.E. and Montgomery, M.T., Alignment of hurricane-like vortices on $f$ and $\beta$ planes. J. Atmos. Sci., 2009, 66, 1779-1792.

Martinsen-Burrell, N., Julien, K., Peterson, M.R. and Weiss, J.B., Merger and alignment in a reduced model for three-dimensional quasigeostrophic ellipsoidal vortices. Phys. Fluids, 2006, 18, 057101.

McKiver, W.J. and Dritschel, D.G., The stability of a quasi- geostrophic ellipsoidal vortex in a background shear flow. J. Fluid. Mech, 2006, 560, 1-17.

McWilliams, J.C., Statistical properties of decaying geostrophic turbulence. J. Fluid Mech., 1989, 198, 199-230.

McWilliams, J.C. and Weiss, J.B., Anisotropic geophysical vortices. Chaos, 1994, 4, 305-311.

Melander, M.V., Zabusky, N.J. and McWilliams, J.C., Symmetric vortex merger in two dimensions: causes and conditions. J. Fluid Mech., 1988, 195, 303-340.

Miyazaki, T., Furuichi, Y. and Takahaski, N., Quasigeostrophic Ellipsoidal Vortex Model. J. Phys. Soc. Jpn., 2001, 70, 1942-1953.

Nof, D. and Dewar, W.K., Alignment of lenses: laboratory and numerical experiments. Deep-Sea Res., 1994, 41, 1207-1229.

Overman II, E.A. and Zabusky, N.J., Evolution and merger of isolated vortex structures. Phys. Fluids, 1982, 25, 1297-1305.

Özuğurlu, E., Reinaud, J.N. and Dritschel, D.G., Interaction between two quasi-geostrophic vortices of unequal potential-vorticity. J. Fluid Mech., 2008, 597, 395-414.

Perrot, X., Carton, X. and Guillou, A., Geostrophic vortex alignment in external shear or strain; in Symposium on Turbulence in the Atmosphere and Oceans, edited by D.G. Dritschel, Vol. 28, 2010, pp. 217-228.

Polvani, L.M., Two-layer geostrophic vortex dynamics. Part 2. Alignment and two-layer V-states. J. Fluid 
Mech., 1991, 225, 241-270

Reasor, P.D. and Montgomery, M.T., Three-dimensional alignment and corotation of weak, TC-like vortices via linear vortex Rossby waves. J. Atmos. Sci., 2001, 58, 2306-2330.

Reasor, P.D. and Montgomery, M.T., Evaluation of a heuristic model for tropical cyclone resilience. J. Atmos. Sci., 2015, 72, 1765-1782.

Reasor, P.D., Montgomery, M.T. and Grasso, L.D., A new look at the problem of tropical cyclones in vertical shear flow: Vortex resiliency. J. Atmos. Sci., 2004, 61, 3-22.

Reinaud, J.N., Three-dimensional quasi-geostrophic vortex equilibria with m-fold symmetry. J. Fluid Mech., 2019, 863, 32-59.

Reinaud, J.N. and Dritschel, D.G., The merger of vertically offset quasi-geostrophic vortices. J. Fluid Mech., 2002, 469, 297-315.

Reinaud, J.N. and Dritschel, D.G., The critical merger distance between two co-rotating quasi-geostrophic vortices. J. Fluid Mech., 2005, 522, 357-381.

Reinaud, J.N., Dritschel, D.G. and Koudella, C.R., The shape of vortices in quasi-geostrophic turbulence. J. Fluid Mech., 2003, 474, 175-192.

Saffman, P.G. and Szeto, R., Equilibrium shapes of a pair of equal uniform vortices. Phys. Fluids, 1980, 23, 2339-2342.

Schecter, D.A., Montgomery, M.T. and Reasor, P.D., A Theory for the Vertical Alignment of a Quasigeostrophic Vortex. J. Atmos. Sci, 2002, 59, 150-168.

Sokolovskiy, M.A. and Carton, X., Baroclinic multipole formation from heton interaction. Fluid Dyn. Res., 2010, 42, 045501.

Sokolovskiy, M.A., Carton, X.J., Filyushkin, B.N. and Yakovenko, O.I., Interaction between a surface jet and subsurface vortices in a three-layer quasi-geostrophic model. Geophys. Astrophys. Fluid Dyn., 2016, 110, 201-223.

Sokolvskiy, M.A. and Verron, J., Dynamics of vortex structures in a stratified rotating fluid, Vol. 47, Atmospheric and Oceanographic Sciences Library 2014 (Heildelberg New York Dordrecht London: Springer).

Sutyrin, G.G., McWilliams, J.C. and Saranavan, R., Co-rotating stationary states and vertical alignment of geostrophic vortices with thin cores. J. Fluid Mech., 1998, 357, 321-349.

Tanahashi, M., Iwase, S. and Miyauchi, T., Appearance and alignment with strain rate of coherent fine scale eddies in turbulent mixing layer. J. Turbulence, 2001, 2, 1-17.

Tychensky, A. and Carton, X., Hydrological and dynamical characterization of Meddies in the Azores region: A paradigm for baroclinic vortex dynamics. J. Geophys. Res., 1998, 103, 25061-25079.

Viera, F., On the alignment and axisymmetrization of a vertically-tilted geostrophic vortex. J. Fluid Mech., 1995, 298, 29-50.

Viúdez, A., Vertical splitting of vortices in geophysical dipoles. J. Phys. Oceanogr., 2010, 40, 2170-2179.

von Hardenberg, J., McWilliams, J.C., Provenzale, A., Shchpetkin, A. and Weiss, J.B., Vortex merging in quasi-geostrohic flows. J. Fluid Mech., 2000, 412, 331353.

Waugh, D.W., The efficiency of symmetric vortex merger. Phys. Fluids A, 1992, pp. 1745-1758.

Zabusky, N., Hughes, M. and Roberts, K., Contour dynamics for the Euler equations in two dimensions. $J$. Comput. Phys., 1979, 20, 96-106.

Zhang, Z., Wang, W. and Qiu, B., Oceanic mass transport by mesoscale eddies. Science, 2014, 345, 322-324.

\section{Appendix A: Evolution of upright-standing spheroid with $h / r=0.5$ and $\ell_{x}^{0} / r=2$}

In this appendix we present the evolution of two initially upright-standing spheroids of uniform potential vorticity, $q=2 \pi$, and of height-to-width aspect ratio $h / r=0.5$ horizontally separated by $\ell_{x}(t=0) / r=\ell_{x}^{0} / r=2$. Figure A1 shows snapshots of the flow evolution. The evolution of the horizontal distance $\ell_{x}$ between the two main structure together with the trajectories of the vortex centres are given in figure A2. Figures A3 and A4 give the distribution in physical length scale $r_{v}$ of the PV volume and of a measure of the self-energy respectively.

\section{Appendix B: 'Cylindrical' vortices}

In this Appendix, we illustrate the existence of multiple branches of V-states. In particular we determine two branch of $\mathrm{V}$-states for vortices whose horizontal cross-sectional area is uniform. We only illustrate the case $\delta z=0$. The first branch (branch $I$ ) stems from two horizontally infinitely distant $\left(\ell_{x} \rightarrow \infty\right)$ cylinders of uniform PV of aspect ratio half-heightto-radius $h / r=1$. The branch is followed by reducing the distance between the two cylinder, 

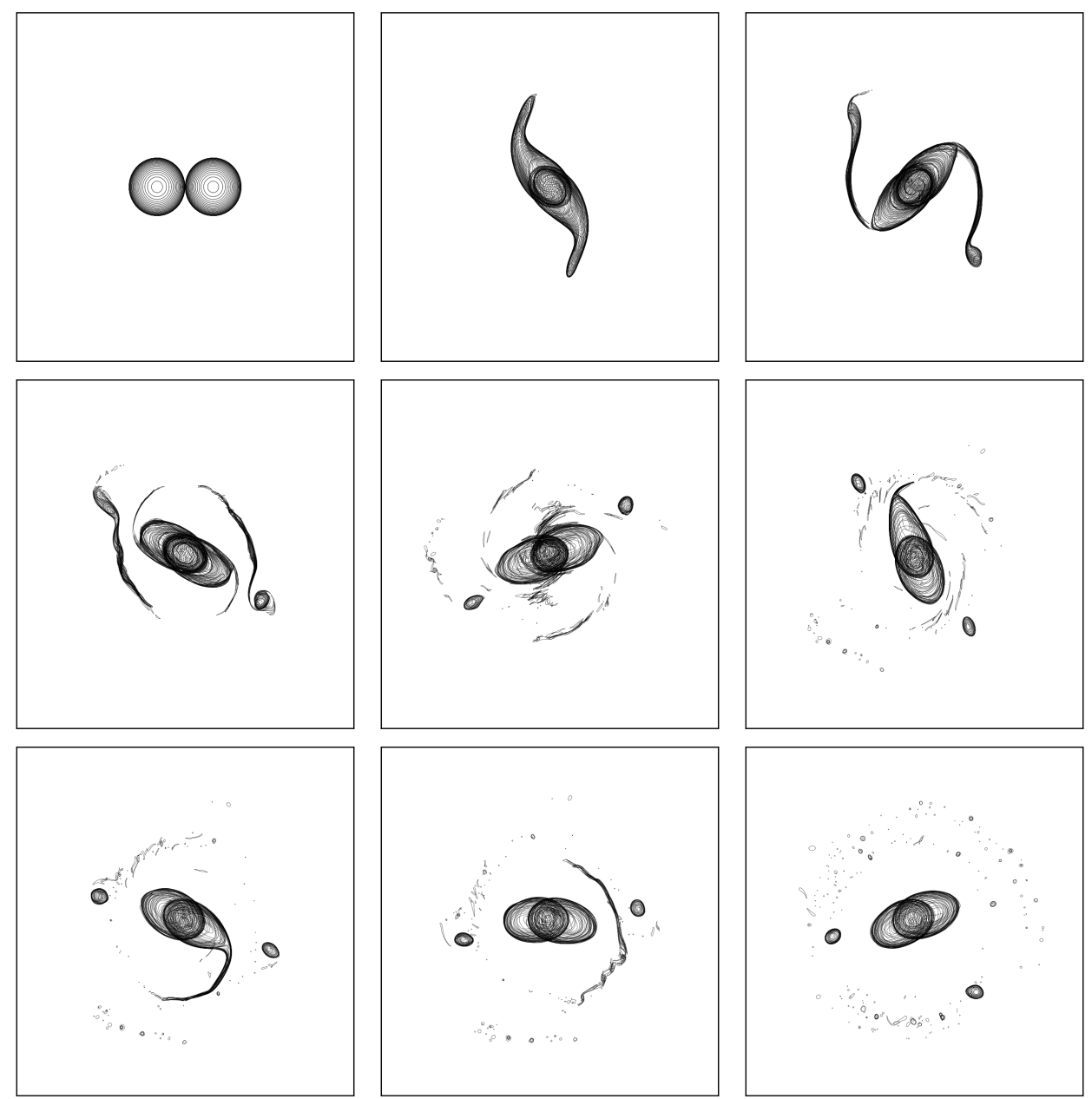

Figure A1. Evolution (full QG dynamics) of a pair of initially upright-standing vortices with $h / r=0.5$ and $\ell_{x} / r=2$. Top view on the vortex bounding contours at $t=0,10,20,40,72,83,92$ and 200.
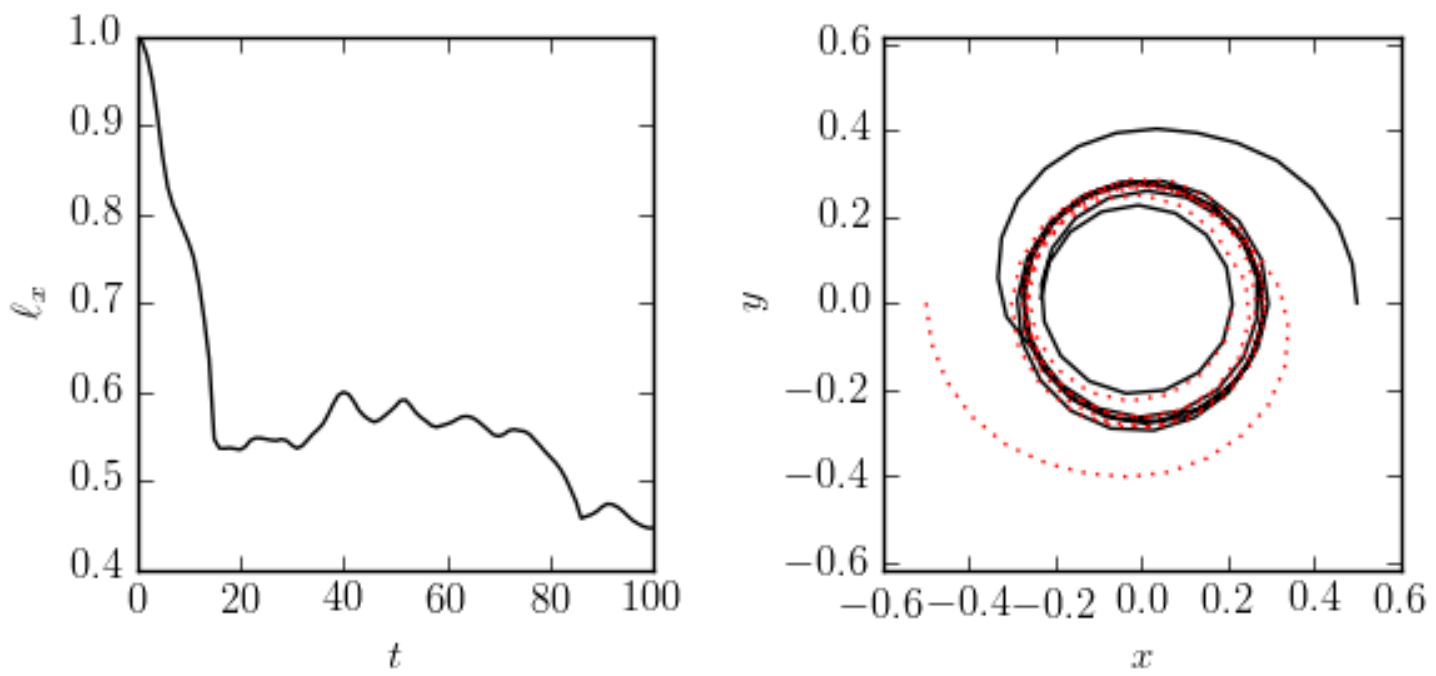

Figure A2. Evolution (full QG dynamics) of the distance between the centres of the two main vortices for of a pair of initially upright-standing vortices with $h / r=0.5$ and $\ell_{x}^{0} / r=2$ (left). Trajectories of the centre of the two main vortices (right). 

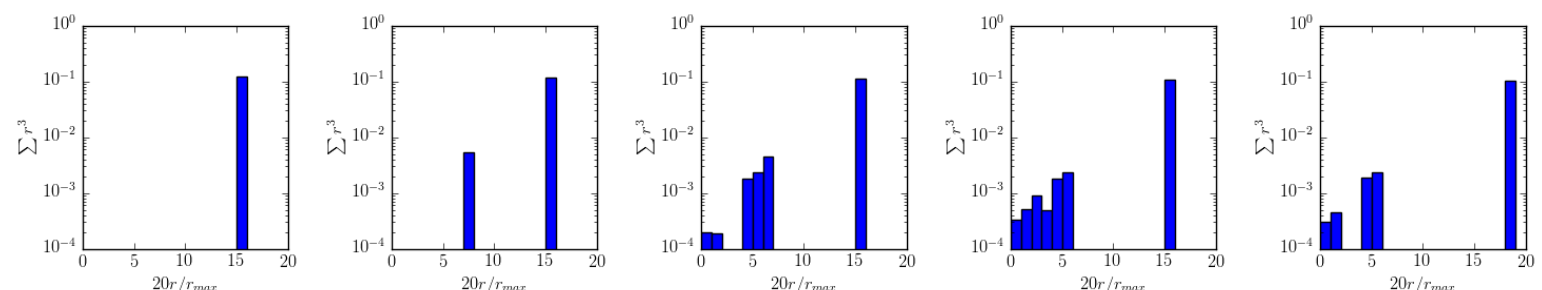

Figure A3. Evolution of a measure of the volume of PV $\sum r_{v}^{3}$ contained in given range of length scale $r_{v}$ for a pair of non-equilibrium, initially upright-standing vortices with $h / r=0.5$ and $\ell_{x}^{0} / r=2$ at $t=0,15,20,40$ and 200 .
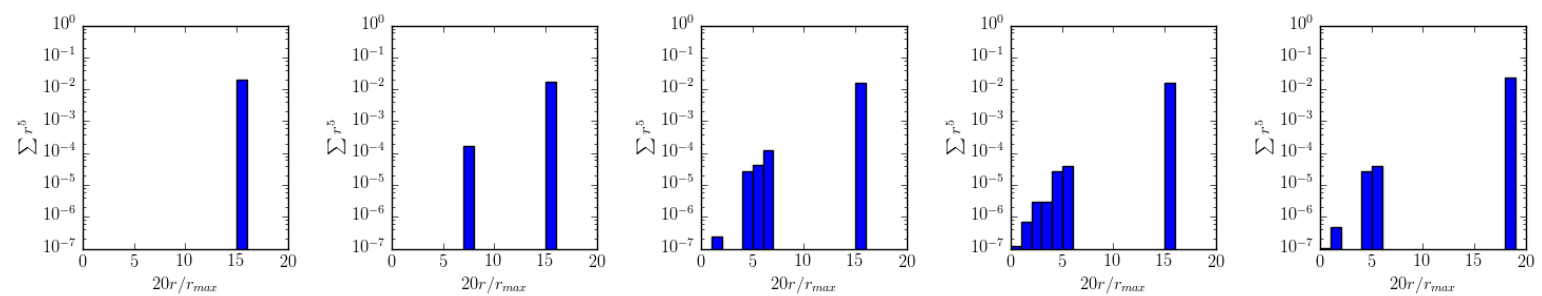

Figure A4. Evolution of an estimate of the self-energy $\sum r_{v}^{5}$ contained in given range of length scale $r_{v}$ for a pair of non-equilibrium, initially upright-standing vortices with $h / r=0.5$ and $\ell_{x}^{0} / r=2$ at $t=0,15,20,40$ and 200 .

namely the horizontal distance between the two outermost edges of the vortices $\Delta x$. The second branch (branch $I I$ ) stems from the two cylinders but aligned $\left(\ell_{x}\right)$. The branch is then followed by increasing the distance between the two outermost edges of the cylinders $\Delta x$. Results are shown in figure B1 for $\Delta x / r=5.6$ and $\Delta x / r=6$. In each case the vortices are explicitly the symmetric image of each other with respect to the centre of the domain.

Branch $I$ ends at the minimum distance $\Delta x / r=5.6$. The horizontal contours bounding the two edges of the vortices where they are the closest form a sharp inner edge (stagnation point) indicating that there are no equilibrium possible along the branch. It should be noted that the vortices are nearly standing upright. For the same value of $\Delta x / r$, the $\mathrm{V}$-state from branch II consists of a single tilted contiguous regions of $\mathrm{PV}$, a tilted cylinder somehow similar to the configuration studied in Reasor and Montgomery (2001) and Schecter et al. (2002) 

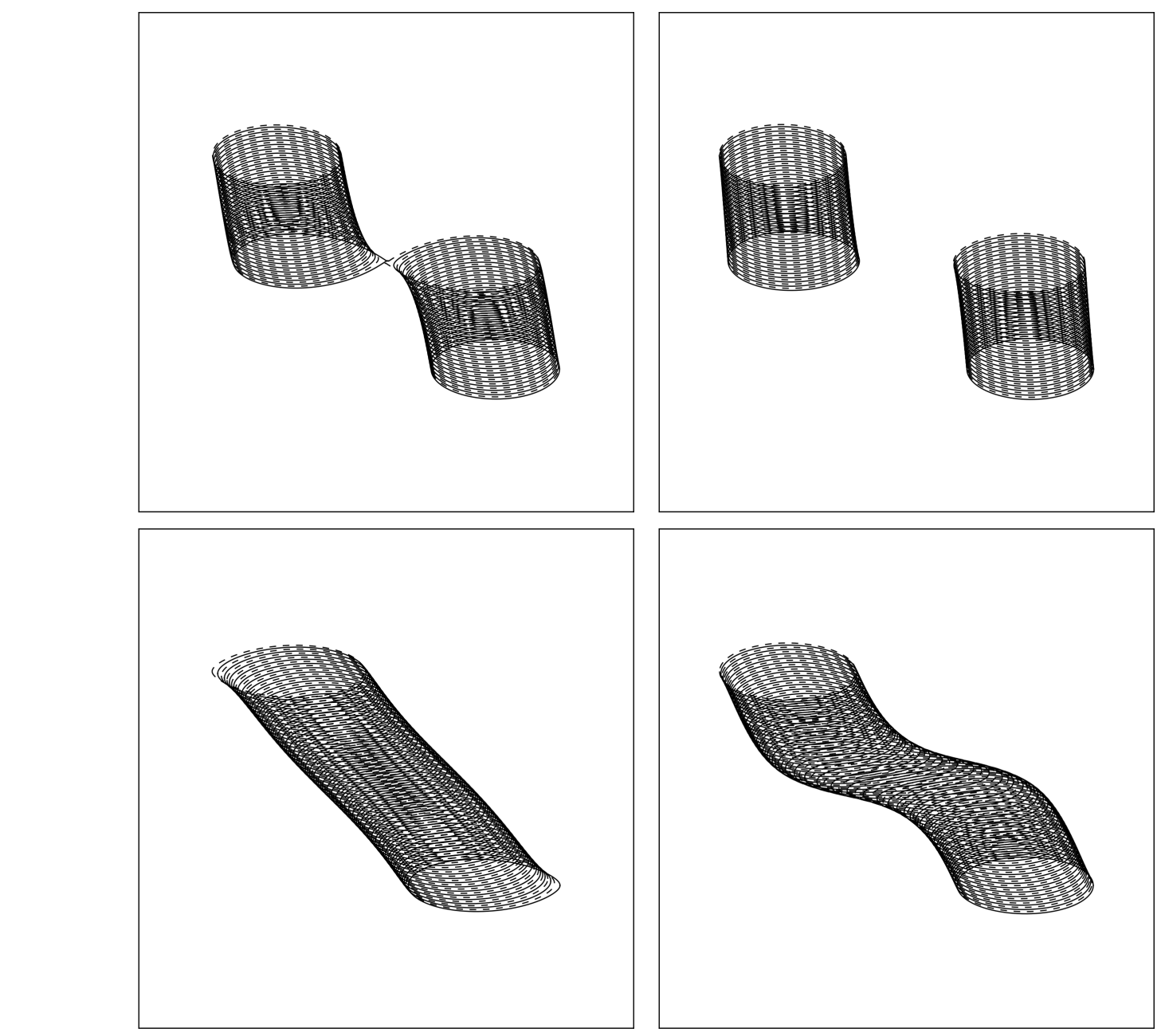

Figure B1. 'Cylindridal' V-states for $h / r=1$ and $\delta z=0$ and $\Delta x / r=5.6$ (left) and $\Delta x / r=6$ (right). Branch $I$ (top row) stemming from $\ell_{x} \rightarrow \infty$ and branch $I I$ (bottom row) stemming from $\ell_{x}=0$.

\footnotetext{
row) steming fiom $\ell_{x} \rightarrow \infty$ and branch $I T\left(\right.$ bottom row) steming fiom $\ell_{x}=0$.
} 